\title{
An Image-Based miRNA Screen Identifies miRNA-135s As Regulators of CNS Axon Growth and Regeneration by Targeting Krüppel-like Factor 4
}

\author{
${ }^{\circ}$ Eljo Y. van Battum, ${ }^{1}$ Marieke G. Verhagen, ${ }^{1}$ Vamshidhar R. Vangoor, ${ }^{1}$ Yuki Fujita, ${ }^{2}$ Alwin A.H.A. Derijck, ${ }^{1}$ \\ ๑Eoghan 0'Duibhir, ${ }^{1}$ Giuliano Giuliani, ${ }^{1}$ Thijs de Gunst, ${ }^{3}$ 'OYouri Adolfs, ${ }^{1}$ Daphne Lelieveld, ${ }^{4}$ David Egan, ${ }^{4}$ \\ ๑Roel Q.J. Schaapveld, ${ }^{3}$-Toshihide Yamashita, ${ }^{2}$ and $\odot$ R. Jeroen Pasterkamp ${ }^{1}$ \\ ${ }^{1}$ Department of Translational Neuroscience, Brain Center Rudolf Magnus, University Medical Center Utrecht, Utrecht University, 3584 CG Utrecht, The \\ Netherlands, ${ }^{2}$ Department of Molecular Neuroscience, Graduate School of Medicine, Osaka University, 2-2 Yamadaoka, Suita, Osaka 565-0871, Japan, \\ ${ }^{3}$ InteRNA Technologies B.V., Yalelaan 62, 3584 CM Utrecht, The Netherlands, and ${ }^{4}$ Department of Cell Biology, Center for Molecular Medicine, University \\ Medical Center Utrecht, Utrecht University, 3584 CX Utrecht, The Netherlands
}

During embryonic development, axons extend over long distances to establish functional connections. In contrast, axon regeneration in the adult mammalian CNS is limited in part by a reduced intrinsic capacity for axon growth. Therefore, insight into the intrinsic control of axon growth may provide new avenues for enhancing CNS regeneration. Here, we performed one of the first miRNome-wide functional miRNA screens to identify miRNAs with robust effects on axon growth. High-content screening identified miR-135a and miR-135b as potent stimulators of axon growth and cortical neuron migration in vitro and in vivo in male and female mice. Intriguingly, both of these developmental effects of miR-135s relied in part on silencing of Krüppel-like factor 4 (KLF4), a well known intrinsic inhibitor of axon growth and regeneration. These results prompted us to test the effect of miR-135s on axon regeneration after injury. Our results show that intravitreal application of miR-135s facilitates retinal ganglion cell (RGC) axon regeneration after optic nerve injury in adult mice in part by repressing KLF4. In contrast, depletion of miR-135s further reduced RGC axon regeneration. Together, these data identify a novel neuronal role for miR-135s and the miR-135-KLF4 pathway and highlight the potential of miRNAs as tools for enhancing CNS axon regeneration.

Key words: axon growth; intrinsic; KLF4; microRNA; regeneration

Significance Statement

Axon regeneration in the adult mammalian CNS is limited in part by a reduced intrinsic capacity for axon growth. Therefore, insight into the intrinsic control of axon growth may provide new avenues for enhancing regeneration. By performing an miRNome-wide functional screen, our studies identify miR-135s as stimulators of axon growth and neuron migration and show that intravitreal application of these miRNAs facilitates CNS axon regeneration after nerve injury in adult mice. Intriguingly, these developmental and regeneration-promoting effects rely in part on silencing of Krüppel-like factor 4 (KLF4), a well known intrinsic inhibitor of axon regeneration. Our data identify a novel neuronal role for the miR-135-KLF4 pathway and support the idea that miRNAs can be used for enhancing CNS axon regeneration.

\section{Introduction}

During neural circuit development, newly born neurons grow their axons over long distances to establish functional connec- tions. This remarkable capacity for axon growth reduces dramatically as the mammalian nervous system matures. Although adult neurons that contribute their axons to peripheral nerves display varying degrees of regrowth after trauma or injury, axonal projections in the mammalian CNS show limited capacity for regeneration. This regenerative failure is caused by the presence of

INNOVATION-1 Collaborative project Epi-miRNA, all to R.J.P., and a Grant-in-Aid for Scientific Research (S) from the Japan Society for the Promotion of Science (25221309) to T.Y. We thank Marina de Wit for technical assistance and members of the Pasterkamp laboratory for helpful discussions.

R.Q.J.S. is a shareholder in InteRNA Technologies B.V. R.Q.J.S. and T.d.G. are stock option holders in InteRNA Technologies B.V. The remaining authors declare no competing financial interests. 
inhibitory factors at the site of injury and a decrease in intrinsic growth ability of affected neurons (Baldwin and Giger, 2015; He and Jin, 2016). One approach to promote CNS axon regeneration has therefore been to target intrinsic factors that promote or inhibit axon growth, such as phosphate and tensin homolog (PTEN), suppressor of cytokine signaling 3 (Socs3), or Krüppel-like factors (KLFs) (He and Jin, 2016). These studies demonstrate that enhancing the intrinsic growth ability of injured neurons induces robust axon regeneration and support the idea that further knowledge of the intrinsic control of axon growth is needed for the design of more effective therapeutic strategies for promoting CNS axon regeneration.

miRNAs are small, noncoding RNAs that regulate gene expression posttranscriptionally by controlling mRNA stability or translation (Lagos-Quintana et al., 2001). miRNAs are highly abundant in the nervous system and have been implicated in various aspects of neuron development and function, including axon growth, guidance, and branching (Baudet et al., 2013; Aksoy-Aksel et al., 2014). However, the in vivo function(s) and downstream mRNA targets of many of the neuronally expressed miRNAs remain unknown. The expression of miRNAs is strongly regulated after nervous system injury and genetic inactivation of miRNA biogenesis hampers peripheral nerve regeneration (Song et al., 2012; Wu et al., 2012; Zou et al., 2013; Hancock et al., 2014; Phay et al., 2015; Li et al., 2016; Martirosyan et al., 2016). In addition, manipulation of miRNA expression in adult sensory neurons promotes peripheral nerve regeneration (Jiang et al., 2015; Gaudet et al., 2016; Hu et al., 2016). However, whether manipulation of miRNAs in injured CNS neurons also enhances CNS axon regeneration in mammals and, if so, through which mRNA targets is unknown.

Here, we performed a genome-wide miRNA screen aimed at identifying functional miRNAs with positive effects on axon growth. High-content screening of $>1000$ miRNAs in neuronal cells identified a novel role for miR-135b and miR-135a as axon growth- and neuron migration-promoting factors. Further, our data show that intravitreal application of miR-135s facilitates regeneration of retinal ganglion cell (RGC) axons after optic nerve injury $(\mathrm{ONI})$ in adult mice. In contrast, decreasing the availability of miR-135s further inhibits RGC axon regeneration. The developmental and regeneration-promoting effects of miR-135s both rely in part on silencing of KLF4, a well known intrinsic inhibitor of axon regeneration (Moore et al., 2009; Qin et al., 2013). Together, these data identify novel neuronal functions for miR-135s and highlight the potential of applying miRNAs to injured CNS neurons as an approach for facilitating CNS axon regeneration.

\section{Materials and Methods}

Animals. All animal use and care was performed in accordance with institutional guidelines and approved by the local ethical animal experimentation committee (Dier Experimenten Commissie; DEC [in Dutch]). C57BL/6J mice (RRID:IMSR_JAX:000664, male and female) were obtained from Charles River Laboratories. When timed-pregnant females were used, the morning on which a vaginal plug was detected was considered embryonic day 0.5 (E0.5). For pups, the day of birth was considered postnatal day 0 (P0).

Lentiviral human whole miRNome library high-content screen and hit confirmation. SH-SY5Y cells, obtained from DSMZ (Acc 209, RRID:

Correspondence should be addressed to Prof. R.J. Pasterkamp, Department of Translational Neuroscience, Brain Center Rudolf Magnus, University Medical Center Utrecht, Universiteitsweg 100, 3584 CG Utrecht, The Netherlands. E-mail: r.j.pasterkamp@umcutrecht.nl.

DOI:10.1523/JNEUROSCI.0662-17.2017

Copyright $\odot 2018$ the authors $\quad 0270-6474 / 18 / 380614-18 \$ 15.00 / 0$
CVCL_0019), were grown in DMEM-F12 (Invitrogen) $+10 \%$ FCS + L-glutamine + penicillin/streptomycin and used between passage 12 and 21. Cells were seeded in 96-well plates using an automated cell seeder Multidrop Combi Reagent Dispenser (Thermo Scientific) at 6000 cells/ well. One day after seeding, cells were treated with $60 \mu \mathrm{M}$ retinoic acid and transduced with a lentiviral human genome-wide miRNA library at an average of $7.34 \times 10^{5}$ IFUs/well (infectious units per well) (InteRNA Technologies). Each library plate was evaluated in triplicate. The lentiviral library contains 640 annotated human miRNA genes (miRBase 12) and 400 candidate miRNAs from deep-sequencing efforts and is based on the pCDH-CMV-MCS-EF1-Puro vector (CD510B-1, System Biosciences; Poell et al., 2011). Systems Biosciences performed the lentiviral packaging and the library had an average of $1.22 \times 10^{9} \mathrm{IFUs} / \mathrm{ml}$. The library was stored in 14 96-well plates. At $4 \mathrm{~d}$ in vitro (DIV), cells were fixed by the addition of 1:1 8\% paraformaldehyde in PBS and blocked in $0.4 \%$ Triton X-100, $5 \%$ goat serum, $1 \%$ BSA, $1 \%$ glycin, and $0.1 \%$ lysin in PBS. Cells were immunostained for $\beta$ III-tubulin (1:3000, mouse monoclonal T8660, Sigma-Aldrich, RRID:AB_477590) with an Alexa Fluor 488-conjugated secondary antibody (Invitrogen) and counterstained with DAPI. Cells were automatically washed thoroughly by two washing cycles with an AquaMax 2000 (Molecular Devices). Automated microscopy was performed using an ArrayScan VTI HCS Reader (Thermo Scientific) and morphological features were extracted with the Cellomics Neuronal Profiling V3 Bioapplication algorithm (settings can be found in Table 1). Raw data (.mdb files) were converted into Excel format using a custom script (courtesy of Ronald van Kesteren, Vrije Universiteit, Amsterdam). All wells with a valid nucleus count $<100$ were removed. Non-neuronal attributes and attributes dependent on cell number were trimmed from the dataset. For all other attributes (as described in Table 1), the plate median was calculated. Each attribute of each well was scored binary ( 0 or 1 ), with a positive score (1) when deviating $>2$ times from the SD of the control median. The median of all miRNAs was used as a control, assuming that most miRNAs would not affect cell morphology. Triplicates of each plate were combined and a well attribute was taken as "true" when a minimum of two of three plates scored positive. This resulted in a final (cumulative) "hitscore," which was used to rank the lentiviral clones with effects on neuronal morphology.

For hit confirmation, SH-SY5Y cells were harvested by trypsinization, washed with PBS, and resuspended at $8 \times 10^{6}$ cells $/ \mathrm{ml}$ in INB buffer containing the following (in $\mathrm{mm}$ ): $135 \mathrm{KCl}, 0.2 \mathrm{CaCl}_{2}, 2 \mathrm{MgCl}_{2}, 10$ HEPES, and 5 EGTA, pH 7.3. Then, cells were mixed with 20 pmol of miRIDIAN mimic [always the human (hsa) isoform; Dharmacon] and electroporated with $3120 \mathrm{~V}$ pulses of $900 \mu$ s and a $2 \mathrm{~s}$ pulse interval in a $1 \mathrm{~mm}$ gap size cuvet in an ECM 830 square wave generator with PEP cuvette module (all BTX; Harvard Apparatus). In this way, $>98 \%$ of the cells are electroporated. Each electroporation was divided and equally distributed $>4$ wells of a 24 -well plate, leaving the outer left and right wells without cells to take into account possible edge-well effects. One day after electroporation, cells were treated with $60 \mu \mathrm{m}$ retinoic acid to induce the development of neuron-like features. Four days after electroporation, cells were fixed and immunostained as described above. Analysis of morphological cell features was performed using the Cellomics software outlined above.

Locked nucleic acid (LNA) in situ hybridization. E16.5 C57BL/6J mouse embryos were collected and decapitated. Brains were fixed in $4 \%$ PFA in PBS and cryoprotected in 30\% sucrose in PBS. Twenty-micrometerthick coronal brain cryosections were made. LNA in situ hybridization was performed as described previously (Kan et al., 2012). Briefly, sections were air-dried and postfixed for $10 \mathrm{~min}$ in $4 \%$ PFA, acetylated for $10 \mathrm{~min}$ at room temperature (RT), treated with proteinase $\mathrm{K}$ at $5 \mu \mathrm{g} / \mathrm{ml}$ for $5 \mathrm{~min}$ at RT, and prehybridized for $1 \mathrm{~h}$ at RT and for $30 \mathrm{~min}$ at $55^{\circ} \mathrm{C}$ before incubation with $15 \mathrm{~nm}$ LNA-containing, double DIG-labeled miR-135a, miR-135b, or control in situ probes (Exiqon) for $2 \mathrm{~h}$ at $55^{\circ} \mathrm{C}$. After hybridization, slides were washed in $0.2 \times$ SSC (saline-sodium citrate) for $1 \mathrm{~h}$ at $55^{\circ} \mathrm{C}$. Slides were blocked for $1 \mathrm{~h}$ with $10 \%$ FCS in PBS and incubated with anti-digoxigenin-AP Fab fragments (1:2500; Roche Diagnostics) in blocking buffer overnight at $4^{\circ} \mathrm{C}$. After PBS washes, slides were incubated with nitroblue and 5-bromo-4-chloro-3-indolyl phosphate (NBT/BCIP, Roche Diagnostics) substrates for 2-20 h at RT. Stain- 
Table 1. Microscope settings and neuronal profiling algorithm parameters

\begin{tabular}{ll}
\hline Microscope settings & \\
\hline Image acquisition & $10 \mathrm{x}$ \\
Objective & ORCA-ER; 1.00 \\
Camera name & Standard $(1024 \times 1024 ; 2 \times 2)$ \\
Acquisition camera mode & AutoFocus $(1024 \times 1024 ; 4 \times 4)$ \\
Autofocus camera mode & 0 \\
Autofocus field interval & \\
Autofocus parameters & 17.6 \\
Fine focus step size & 9 \\
Fine focus plane count & 70.4 \\
Coarse focus step size & 9 \\
Coarse focus plane count & 21 \\
Smart focus plane count & False \\
Use extended range focusing & False \\
Apply backlash correction & STANDARD \\
Autofocus method & False \\
Use relaxed pass/fail criteria & 0 \\
Focus edge threshold & 0 \\
Focus adjustment & 0.2 \\
Focus score min ratio & 0.4 \\
Focus score mid ratio & 0.5 \\
Focus score max ratio & 0.1 \\
Focus exposure time for auto-expose (s) & \\
Scan limits & 10 \\
Max fields for well & No Limit \\
Min objects for well & No Limit \\
Max sparse fields for well & N/A A \\
Min objects for field & \\
Max sparse wells for plate & \\
\hline
\end{tabular}

\begin{tabular}{|c|c|c|}
\hline \multicolumn{3}{|l|}{ Channel 1: nucleus } \\
\hline Dye & \multicolumn{2}{|c|}{ XF100 - Hoechst } \\
\hline Apply illumination correction & \multicolumn{2}{|c|}{ False } \\
\hline Apply background correction & \multicolumn{2}{|c|}{ True } \\
\hline Gain & \multicolumn{2}{|c|}{25} \\
\hline Use apotome & \multicolumn{2}{|c|}{ False } \\
\hline Z offset & \multicolumn{2}{|c|}{0.00} \\
\hline \multicolumn{3}{|l|}{ Exposure parameters (Ch 1$)$} \\
\hline Method & \multirow{2}{*}{\multicolumn{2}{|c|}{$\begin{array}{l}\text { Fixed } \\
0.495051\end{array}$}} \\
\hline Exposure time (s) & & \\
\hline \multicolumn{3}{|l|}{ Object identification (Ch 1 ) } \\
\hline Method & \multirow{2}{*}{\multicolumn{2}{|c|}{$\begin{array}{l}\text { TriangleThreshold } \\
0\end{array}$}} \\
\hline Value & & \\
\hline Object selection parameter & Min & Max \\
\hline NucAreaCh1 & 50 & 400 \\
\hline NucShapeP2ACh1 & 0 & 1000 \\
\hline NucShapeLWRCh1 & 0 & 10 \\
\hline NucAvgIntenCh1 & 0 & 4095 \\
\hline NucTotallntenCh1 & 0 & 10000000000 \\
\hline NucVarlntenCh1 & 0 & 32767 \\
\hline \multicolumn{3}{|l|}{ Display options (Ch 1) } \\
\hline \multicolumn{3}{|l|}{ Composite color (Hex) \#0000FF } \\
\hline \multicolumn{3}{|l|}{ ValidNucleus \#0000FF } \\
\hline \multicolumn{3}{|l|}{ RejectedNucleus \#FF7F00 } \\
\hline \multicolumn{3}{|l|}{ Channel 2: neuronal } \\
\hline Dye & & XF100 - FITC (GFP) \\
\hline Apply illumination correction & & False \\
\hline Apply background correction & & True \\
\hline Gain & & 25 \\
\hline Use apotome & & False \\
\hline Z offset & & 0.00 \\
\hline \multicolumn{3}{|l|}{ Exposure parameters (Ch 2$)$} \\
\hline Method & & Fixed \\
\hline Exposure time (s) & & $\begin{array}{l}0.301046 \\
\text { (Table continues) }\end{array}$ \\
\hline
\end{tabular}

Table 1. Continued

\begin{tabular}{lcr}
\hline Microscope settings & \multicolumn{2}{l}{} \\
\hline $\begin{array}{l}\text { Object identification (Ch 2) } \\
\text { Method }\end{array}$ & \multicolumn{1}{l}{ IsodataThreshold } \\
Value & 0 & Max \\
Object selection parameter & Min & 100000 \\
\hline NeuriteLengthCh2 & 0 & 1000 \\
NeuriteWidthCh2 & 0 & 4095 \\
NeuriteAvglntenCh2 & 0 & 10000000000 \\
NeuriteTotallntenCh2 & 1 & 1 \\
CellBodyNucCountCh2 & 0 & 250000 \\
CellBodyAreaCh2 & 0 & 10 \\
CellBodyShapeP2ACh2 & 0 & 10 \\
CellBodyShapeLWRCh2 & 0 & 4095 \\
CellBodyAvglntenCh2 & 0 & 10000000000 \\
CellBodyTotallntenCh2 & & \\
Display options (Ch 2) & & \\
Composite color (Hex) \#00FF40 & & \\
Neurite \#FF00FF & & \\
Neurite \#00FF00 & & \\
SelectedCellBody \#0080FF & & \\
RejectedCellBody \#FF0000 & & \\
NeuritePoint \#FFFF00 & & \\
Assay algorithm neuronal profiling & V3.5 \\
Assay version & 6.2 (Local Version: 6.20.0.3519) \\
Focus channel & 1 & \\
No. of channels & 2 & \\
\hline
\end{tabular}

\section{Assay parameters for hit score}

UseReferenceWells

MinRefAvgNeuronCountPerField

UseMicrometers

PixelSize

Type_1_EventDefinition

Type_2_EventDefinition

Type_3_EventDefinition

NucTypeCh1

BackgroundCorrectionCh1

NucSmoothFactorCh1

RejectBorderNucsCh1

CellBodyNucTotalAreaCh1LevelHigh

CellBodyNucTotalAreaCh1LevelHigh_CC

CellBodyNucTotallntenCh1LevelHigh

CellBodynucTotallntenCh1LevelHigh_CC

CellBodyNucAvgIntenCh1LevelHigh

CellBodyNucAvgIntenCh1LevelHigh_CC

MinCellBodyNucOverlapCh2

CellBodyAndNeuriteTypeCh2

BackgroundCorrectionCh2

CellBodySmoothFactorCh2

CellBodyDemarcationCh2

UseNucForCellBodySegmentationCh2

CellBodyMaskModifierCh2

RejectBorderCellBodiesCh2

NeuriteSmoothFactorCh2

NeuriteldentificationModifierCh2

NeuriteDetectRadiusCh2

NeuriteDetectMethodCh2

NeuriteDirectionCh2

NeuritePointResolutionCh2

NeuritePointDisplayModeCh2

RejectMultiplyTracedNeuritesCh2

UseCellBodyZOIForNeuriteTracingCh2

CellBodyNucCountCh2LevelHigh
1

1.29

90324.03

0

0

0

$-1$

0

1

110

80000

1

850

1

0

0

2

1

1

1

$-0.97$

3

4

3

1

2

0

0 
Table 1. Continued

\begin{tabular}{|c|c|}
\hline Assay parameters & \\
\hline CellBodyNucCountCh2LevelHigh_CC & 1 \\
\hline CellBodyAreaCh2LevelHigh & 340 \\
\hline CellBodyAreaCh2LevelHigh_CC & 1 \\
\hline CellBodyShapeP2ACh2LevelHigh & 1.3 \\
\hline CellBodyShapeP2ACh2LevelHigh_CC & 1 \\
\hline CellBodyShapeLWRCh2LevelHigh & 1.6 \\
\hline CellBodyShapeLWRCh2LevelHigh_CC & 1 \\
\hline CellBodyTotallntenCh2LevelHigh & 150000 \\
\hline CellBodyTotallntenCh2LevelHigh_CC & 1 \\
\hline CellBodyAvgIntenCh2LevelHigh & 650 \\
\hline CellBodyAvgIntenCh2LevelHigh_CC & 1 \\
\hline NeuriteTotalCountCh2LevelHigh & 3 \\
\hline NeuriteTotalCountCh2LevelHigh_CC & 1 \\
\hline NeuriteTotallengthCh2LevelHigh & 20 \\
\hline NeuriteTotalLengthCh2LevelHigh_CC & 1 \\
\hline NeuriteAvgLengthCh2LevelHigh & 8 \\
\hline NeuriteAvgLengthCh2LevelHigh_CC & 1 \\
\hline NeuriteMaxLengthWithBranchesCh2LevelHigh & 10 \\
\hline NeuriteMaxLengthWithBranchesCh2LevelHigh_CC & 1 \\
\hline NeuriteMaxLengthWithoutBranchesCh2LevelHigh & 10 \\
\hline NeuriteMaxLengthWithoutBranchesCh2Level-High_CC & 1 \\
\hline NeuriteTotalAreaCh2LevelHigh & 60 \\
\hline NeuriteTotalAreaCh2LevelHigh_CC & 1 \\
\hline NeuriteWidthCh2LevelHigh & 3.57 \\
\hline NeuriteWidthCh2LevelHigh_CC & 1 \\
\hline NeuriteTotallntenCh2LevelHigh & 8000 \\
\hline NeuriteTotallntenCh2LevelHigh_CC & 1 \\
\hline NeuriteAvgIntenCh2LevelHigh & 200 \\
\hline NeuriteAvgIntenCh2LevelHigh_CC & 1 \\
\hline NeuriteVarlntenCh2LevelHigh & 125 \\
\hline NeuriteVarIntenCh2LevelHigh_CC & 1 \\
\hline BranchPointTotalCountCh2LevelHigh & 0.2 \\
\hline BranchPointTotalCountCh2LevelHigh_CC & 1 \\
\hline BranchPointAvgCountCh2LevelHigh & 0.1 \\
\hline BranchPointAvgCountCh2LevelHigh_CC & 1 \\
\hline BranchPointCountPerNeuriteLengthCh2LevelHigh & 0 \\
\hline BranchPointCountPerNeuriteLengthCh2Level-High_CC & 1 \\
\hline BranchPointAvgDistFromCellBodyCh2LevelHigh & 1 \\
\hline BranchPointAvgDistFromCellBodyCh2Level-High_CC & 1 \\
\hline CrossPointTotalCountCh2LevelHigh & 0.1 \\
\hline CrossPointTotalCountCh2LevelHigh_CC & 1 \\
\hline CrossPointAvgCountCh2LevelHigh & 0.1 \\
\hline CrossPointAvgCountCh2LevelHigh_CC & 1 \\
\hline NucSegmentationCh1 & 4.1 \\
\hline CellBodySegmentationCh2 & 14.1 \\
\hline
\end{tabular}

Cellomics Arrayscan microscope settings are described in the text section "Image acquisition." Nuclear DAPI staining was detected in channel 1 , whereas the cell body and neurites stained by $\beta 3$-tubulin were detected in channel 2 . The neuronal profiling algorithm recognition parameters of the cells are described in the text sections "Channel 1: nucleus" and "Channel 2: neuronal." In the text section "Assay parameters," parameters describing general neuron morphology, such as the number of neurites, neurite length, number of branch points, are listed. The effect of each miRNA on these parameters was scored as described in the Materials and Methods and in Figure $1 A$.

ing was terminated by washing of the slides in PBS. Slides were mounted in $90 \%$ glycerol in PBS. Sections stained with scrambled LNA-DIG probe were devoid of specific staining.

$q P C R$. E14.5 and E16.5 C57BL/6 embryos, P0 and P10 pups, and adult mice were decapitated and brains were removed. Hippocampi and cortices were dissected and frozen immediately in dry ice. Total RNA was isolated from at least three animals from three different litters using the miRNeasy kit (Qiagen) according to the manufacturer's protocol. In addition, total RNA was isolated from primary hippocampal neurons from 3-4 coverslips of 2 different cultures at DIV2, DIV7, DIV14, and DIV21. Furthermore, total RNA was isolated from retinas $14 \mathrm{~d}$ after ONI experiments and intravitreal injection of miRNA mimics (see paragraph describing ONI experiments). RNA quantity was determined using Nanodrop (Thermo Scientific) and equal amounts of each sample were used for first-strand cDNA synthesis using a universal cDNA synthesis kit
(Exiqon). qPCRs were run on Quantstudio 6 Flex Real-Time PCR system (Applied Biosystems) using miRNA LNA PCR primer sets and SYBR Green master mix (Exiqon). All samples were run in duplicate. Ct values were determined using QuantStudio Real-Time PCR software version 1.1. The expression levels of different miRNAs were estimated by normalization to $5 \mathrm{~S}$ rRNA and the statistical significance was analyzed with single-factor ANOVA. $p<0.05$ was considered significant.

Culturing and transfection of mouse hippocampal and cortical neurons. Hippocampal and cortical cultures were generated as described previously (Van Battum et al., 2014). In brief, P0-P1 C57BL/6 mouse pups were decapitated and brains were rapidly removed in ice-cold dissection medium. Hippocampi or cortices were isolated, trypsinized, and dissociated into single cells and then cultured in neurobasal medium supplemented with B-27, L-glutamine, penicillin/streptomycin, and $\beta$-mercaptoethanol on acid-washed poly-D-lysine- $(20 \mu \mathrm{g} / \mathrm{ml})$ and laminin $(40 \mu \mathrm{g} / \mathrm{ml})$-coated glass coverslips at $37^{\circ} \mathrm{C}+5 \% \mathrm{CO}_{2}$ in 12 -well plates. On DIV1, neurons were cotransfected with $0.5 \mu \mathrm{g}$ of CAG-GFP vector and $50 \mathrm{pmol}$ of miRIDIAN mimics for miR-135a, miR-135b, or control-1 mimic (also known as Negative control-A; Dharmacon) per well or $0.5 \mu \mathrm{g}$ of miRNA H1mCherry-sponge vectors per well for miR-135a or miR-135b (Tebu-Bio) using Lipofectamine 2000 (Invitrogen). For rescue experiments, a pCMV-KLF4-EGFP vector (Origene) was used. On DIV4, neurons were fixed with $4 \%$ PFA and $4 \%$ sucrose in PBS. For immunocytochemistry, neurons were incubated with rabbit anti-GFP (1:1000, A-11122, Invitrogen, RRID:AB_221569) or rabbit-anti-RFP (1:1000, Rockland, RRID: AB_11182807) and mouse anti- $\beta$ III tubulin (1:3000, T8660, SigmaAldrich, RRID:AB_477590) dissolved in 3\% normal horse serum, $0.1 \%$ BSA, and $0.1 \%$ Triton X-100 in PBS. Images were taken using an Axioskop 2 EPI fluorescent microscope (Zeiss). The longest neurites were traced semimanually using the NeuronJ plugin (RRID:SCR_002074) of ImageJ and Sholl analysis was performed using ImageJ software (RRID: SCR_003070). More than 100 transfected neurons from at least three independent experiments were traced. Nonpaired parametric $t$ tests were performed in Prism version 6 (GraphPad, RRID:SCR_002798) to analyze the data statistically.

miRNA target finding and validation. The miRecords database was used to search for shared mRNA targets of miR-135a and miR-135b predicted by at least six target prediction programs (Xiao et al., 2009).

Predicted targets shared by miR-135a and miR-135b were postselected on the basis of potential involvement in neuronal development. For target validation, the entire 3 '-UTR from KLF4 was retrieved from cDNA and cloned into the psiCHECK2 vector (Promega). PCR-mediated mutagenesis of the KLF4 $3^{\prime}$-UTR was performed to alter the binding site located at $394 \mathrm{nt}$ of the KLF4 3'-UTR (see Fig. 5A, arrow). HEK293 cells (RRID:CVCL_0045) were transfected using Lipofectamine with $250 \mathrm{ng}$ of vector and 20 pmol of miRIDIAN miRNA mimic (Dharmacon). Cells were lysed $24 \mathrm{~h}$ after transfection and examined with a Dual-Luciferase reporter assay (E1960; Promega) on a spectrophotometer. $t$ tests were performed to compare luciferase activity in Prism version 6 software (GraphPad, RRID:SCR_002798).

For protein analysis, miRIDIAN miRNA mimics (Dharmacon) were transfected into Neuro2A cells (ATCC, RRID:CVCL_0470) using Lipofectamine 2000. After $24 \mathrm{~h}$, cells were lysed in lysis buffer $(20 \mathrm{~mm}$ Tris $\mathrm{pH}$ 8.0, $150 \mathrm{~mm} \mathrm{KCL}, 1 \%$ Triton X-100, and protease inhibitor (Roche) in milliQ (MQ). Samples were separated on $8 \%$ SDS-PAGE gels and blotted onto nitrocellulose membrane. Nonspecific binding was blocked with $5 \%$ milk in TBS-Tween for $1 \mathrm{~h}$ at RT. After incubation with rabbit-antiKLF4 (1:500, Santa-Cruz Biotechnology, RRID:AB_669567) and mouseanti- $\beta$-actin (1:5000, Sigma-Aldrich, RRID:AB_476743) in 1\% milk in TBS-Tween, blots were stained with peroxidase-conjugated secondary antibodies (Abcam). Signals were detected using Pierce ECL Western Detection Reagent and images were made using the FluorChem M Imaging system (Protein Simple). ImageJ was used to determine protein levels in the individual bands and KLF4 expression was normalized to $\beta$-actin levels in the same sample. $t$ tests were performed to compare the relative KLF4 expression between conditions (GraphPad Prism version 6 software, RRID:SCR_002798).

Immunohistochemistry. E16.5 C57BL/6J mouse embryos or adult mice were collected and decapitated. Brains were fixed in 4\% PFA in PBS and 
cryoprotected in 30\% sucrose in PBS. Twenty-micrometer-thick coronal brain cryosections were made. Sections were incubated with rabbit antiKLF4 (Santa-Cruz Biotechnology, 1:500, no longer available) or LabNed LN2023880 (1:100, RRID:AB_2687557) diluted in 3\% BSA and 0.1\% Triton X-100 in PBS, stained with Alexa Fluor-conjugated secondary antibody, and counterstained with DAPI. Images were made using an AxioScope EPI fluorescent microscope (Zeiss) and a confocal scanning microscope (Olympus).

Ex vivo electroporation. Ex vivo electroporation was performed as described previously (Yau et al., 2014). In brief, pregnant C57BL/6 mice were killed by cervical dislocation and E14.5 embryos were removed rapidly and decapitated. Then, $30 \mu \mathrm{M}$ miRIDIAN mimics (Dharmacon) for miR-135a, miR-135b, or control-1 combined with $0.4 \mu \mathrm{g} / \mu \mathrm{l}$ pCAGGFP vector were dissolved in $0,1 \%$ Fast Green in MQ and $1.7 \mu \mathrm{l}$ of this mixture was injected in the lateral ventricles using glass micropipettes (Harvard Apparatus) and a microinjector. Heads were subjected to three $100 \mathrm{~ms}$ pulses of $30 \mathrm{~V}$ with $100 \mathrm{~ms}$ pulse interval using gold-plated gene paddle electrodes and an 830 square-wave generator (BTX; Harvard Apparatus). Brains were then isolated, collected in cHBSS, embedded in 3\% LMP-Agarose (Fisher Scientific) in cHBSS, and sectioned coronally into $250-\mu \mathrm{m}$-thick slices using a vibratome (Leica). Sections were collected on poly-D-lysin-laminin-coated culture membrane inserts (Falcon), placed on top of slice culture medium (70\% v/v basal Eagle medium, 26\% v/v cHBSS, $20 \mathrm{~mm}$ D-glucose, $1 \mathrm{~mm}$ L-glutamine, and penicillin/streptomycin) and cultured for $4 \mathrm{~d}$ to assess the degree of migration. Cultures were fixed with $4 \%$ PFA, blocked in $3 \%$ BSA and $0.1 \%$ Triton in PBS, and stained with rabbit anti-GFP (1:1000, A-11122, Invitrogen, RRID: AB_221569) and mouse anti-MAP2 SMI 52 (1:1000, Abcam, RRID: AB_776173) antibodies. Z Z-stack images were taken using a confocal laser-scanning microscope (Olympus). Migration of GFP-positive cells was analyzed as follows: using Adobe Photoshop, consistent rectangles divided in 8 equal bins were placed on top of the image so that bin 1 included the ventricular zone (VZ) and bin 8 covered the marginal zone, as shown schematically in Figure $4 A$. Cells in each bin were counted and divided by the total amount of cells in the rectangle. The average of at least two rectangles of each image was used for comparison. For each condition, 12 cortical slices from at least three different experiments were used. Nonparametric Mann-Whitney $U$ tests were performed in Prism version 6 software (GraphPad, RRID:SCR_002798) to compare migration between control and miRNA overexpression.

In utero electroporation. In utero electroporation was performed as described previously (van Erp et al., 2015). Pregnant C57BL/6 mice at E14.5 were deeply anesthetized with isoflurane (induction: $3-4 \%$, surgery: $1.5-2 \%$ ), injected with $0.05 \mathrm{mg} / \mathrm{kg}$ buprenorfinhydrochloride in saline, and the abdominal cavity was opened under sterile surgical conditions. Uterine horns were exposed and $1.7 \mu$ l of DNA mixture containing $0.4 \mu \mathrm{g} / \mu \mathrm{l}$ pCAG-GFP and $15 \mu \mathrm{M}$ miR-135a and $15 \mu \mathrm{M}$ miR-135b mimic, $30 \mathrm{pmol}$ of control- $1 \mathrm{mimic}, 0.6 \mu \mathrm{g} / \mu \mathrm{l} \mathrm{scrambled} \mathrm{sponge} \mathrm{vector,}$

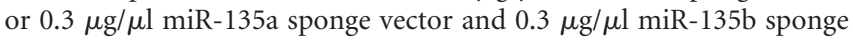
vector (H1-mCherry vectors; Tebu-bio) dissolved in MQ with $0.05 \%$ Fast Green (Sigma-Aldrich) was injected in the lateral ventricles of the embryo's using glass micropipettes (Harvard Apparatus) and a PLI-100 Pico-injector (Harvard Apparatus). For rescue experiments, $0.2 \mu \mathrm{g} / \mu \mathrm{l}$ pCAG-GFP was combined with $0.2 \mu \mathrm{g} / \mu \mathrm{l}$ pCAG-KLF4 and $15 \mu \mathrm{m} \mathrm{miR-}$ $135 \mathrm{a}$ and $15 \mu \mathrm{M}$ miR-135b mimic. Brains were electroporated using an ECM 830 Electro-Square-Porator (Harvard Apparatus) set to five unipolar pulses at $30 \mathrm{~V}$ ( $50 \mathrm{~ms}$ pulse length interval and $950 \mathrm{~ms}$ pulse length). The motor cortex was targeted by holding the head with a platinum tweezer-electrode (negative pole) while a third a gold-plated Genepaddle (positive pole; Fisher Scientific) was placed on top of the head. Embryos were placed back into the abdomen and abdominal muscles and skin were sutured separately. Release from isoflurane awakened the mother mice. Embryos were collected at E16.5 and pups at P4 or P10. Heads were fixed in $4 \%$ PFA in PBS and submerged in 30\% sucrose. Then, $20-\mu \mathrm{m}-$ thick coronal cryosections were made and immunohistochemistry and cortical migration analysis were performed as described for ex vivo electroporated slices. To measure neurite outgrowth in vivo, leading process length was traced using ImageJ. In the case of endogenous miR-135 downregulation, where brains were electroporated with H1-mCherry sponge vectors (Tebu-bio), neuron migration and leading process length were analyzed upon staining with rabbit-anti-RFP (1:1000; Rockland, RRID:AB_11182807). Control and miRNA test conditions were always equally distributed among the embryos in the uterus. Analysis was always performed on the slice in which the corpus callosum was first complete and in one or two consecutive slices. At least five embryos from at least two separate experiments were used for comparison.

ONI and in vivo gene transfection. Three-week-old C57BL/6J mice were obtained from Hamamatsu. ONI was performed as described previously in detail (van Erp et al., 2015). The left optic nerve was crushed with fine forceps for $10 \mathrm{~s} \sim 1 \mathrm{~mm}$ posterior to the optic disc. Then, 50 $\mathrm{pmol} / \mu \mathrm{l} \mathrm{miR}-135 \mathrm{a}$ and $50 \mathrm{pmol} / \mu \mathrm{lmiR}-135 \mathrm{~b}$ or $100 \mathrm{pmol} / \mu \mathrm{l}$ control-1 mimic were injected intravitreally (with lipofectamine) immediately after injury and on day 7 after axotomy. In vivo gene transfection was performed as described previously (van Erp et al., 2015). Briefly, pCAGGFP or pCAG-KLF4 was mixed with miRNA mimics and Lipofectamine 2000. Then, $2 \mu \mathrm{l}$ of the complexes were injected intravitreally immediately after injury and on day 7 after axotomy. Nine mice were used for each group. Similarly, $4 \mu \mathrm{g}$ of sponge vectors specifically targeting miR$135 \mathrm{a}$ and miR-135b or control sponge (Tebu-bio) were injected intravitreally (with lipofectamine). Six mice per group were used. AAV2 virus (AAV-miR-GFP-blank control virus, catalog \#Am00102, GFP mmumiR-135a-5p AAV miRNA virus, catalog \#Amm1006802, GFP mmumiR-135b-5p AAV miRNA virus, catalog \#Amm1007002, abm) was injected at $7 \mathrm{~d}$ before ONI. To visualize RGC axons, $1 \mu \mathrm{l}$ of cholera toxin $\beta$ subunits conjugated to Alexa Fluor 555 ( $2 \mu \mathrm{g} / \mu \mathrm{l}$; Invitrogen) was injected into the vitreous with a glass needle $12 \mathrm{~d}$ after the injury. On day 14 after axotomy, animals were perfused with $4 \%$ PFA. The eye cups with the nerve segment attached were postfixed and immersed in $30 \%$ sucrose overnight at $4^{\circ} \mathrm{C}$. Tissues were embedded in Tissue Tek and serial crosssections $(16 \mu \mathrm{m})$ were prepared using cryostat and collected on MAScoated glass slides (Matsunami). Axonal regeneration was quantified by counting the number of CTB-labeled fibers extending 0.2, 0.5, and 1.0 $\mathrm{mm}$ from the distal end of the lesion site in 5 sections. The cross-sectional width of the optic nerve was measured at the point at which the counts were taken and was used to calculate the number of axons per millimeter of nerve width. The number of axons per millimeter was then averaged over the five sections. $\sum a d$, the total number of axons extending distance $d$ in a nerve having a radius of $r$, was estimated by summing all the sections having a thickness $t(16 \mu \mathrm{m})$ as follows: $\sum a d=\pi r^{2} \times$ [average axons $/ \mathrm{mm}] / t$. Statistical analyses were performed using one-way ANOVAs. $p<0.05$ was considered significant.

Experimental design and statistical analysis. In this study, C57BL/6J mice were used regardless of their sex. For statistical analyses, Prism version 6 software (GraphPad) was used. Generally, unpaired $t$ tests were used to compare the means of two groups, with the exception of neuron migration analyses (nonparametric Mann-Whitney $U$ tests) and qPCR analyses (single-factor ANOVA). For all statistical tests, significance was set at $p<0.05$. Exact $p$ values, $t$-values, and degrees of freedom are provided in the results and $n$ values are provided in the figure legends.

At the start of this study, an automated morphological Cellomics screen of retinoic acid-treated SH-SY5Y cells that were transduced with a lentiviral library containing 1140 unique human miRNAs (Poell et al., 2011) was performed to identify miRNAs that (positively) influence neuronal features (Fig. 1A). The screen was conducted in triplo and morphological parameters were scored with a neuroprofiling algorithm (Table 1). To confirm the effect of the most robust miRNAs, the Cellomics analysis was repeated on SH-SY5Y cells that were electroprated with miRNA mimics for a selection of hits. This experiment was performed three times in quadruplo (i.e., $3 \times 4$ coverslips; Fig. $1 E$ ) and analyzed statistically using Student $t$ tests.

Expression of miR-135a and miR-135b in the mouse brain at different ages was tested by LNA in situ hybridization and qPCR experiments (in tissue of at least three different mice per age (Fig. 2). Expression of miR$135 \mathrm{a}$ and miR-135b was also determined in cultured primary hippocampal neurons (Fig. 3A). qPCR experiments were analyzed statistically using single-factor ANOVAs.

Next, the effect of miR-135a and miR-135b overexpression and downregulation was examined in primary neuron cultures. Lipofectamine-based 


\section{A Cell culture and immunocytochemistry}

Day 1:

SH-SY5Y 6000/well

Retinoic Acid $60 \mu \mathrm{M}$
DIV 1:

Virus library

$7.34 * 10^{5}$ IFU/well
DIV 4:

Fix cells (4\% PFA) Immunocytochemistry ( $\beta$ III-Tubulin, DAPI)

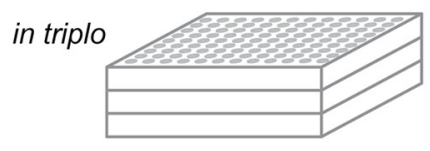

Cellomics Arrayscan

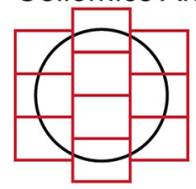

$10 x$ objective 10 fields/well AutoFocus, Fixed exposure

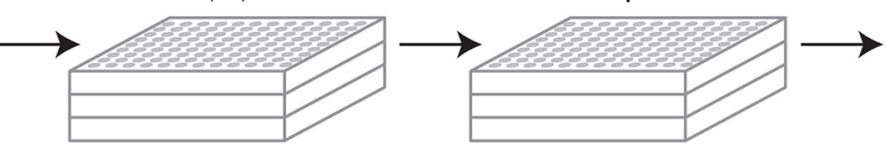

\section{Neuronal profiling algorithm}

binary scoring method: parameter $x>$ median $+2 *$ stdev, true $=1$ false $=0$ true if wells score 1 in $2 / 3$ or $3 / 3$ plates Included if total cell count $>100$ and total hitscore $>6$
B

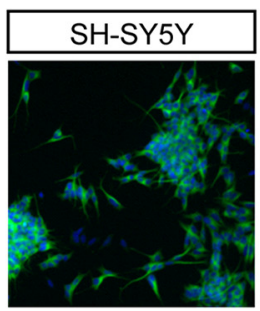

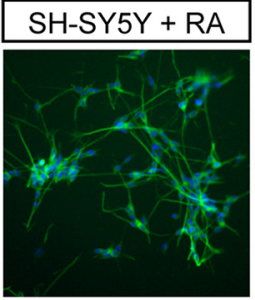

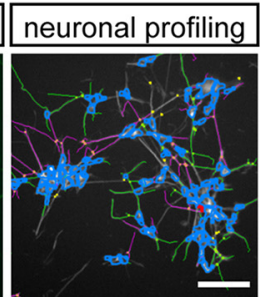

\section{C}

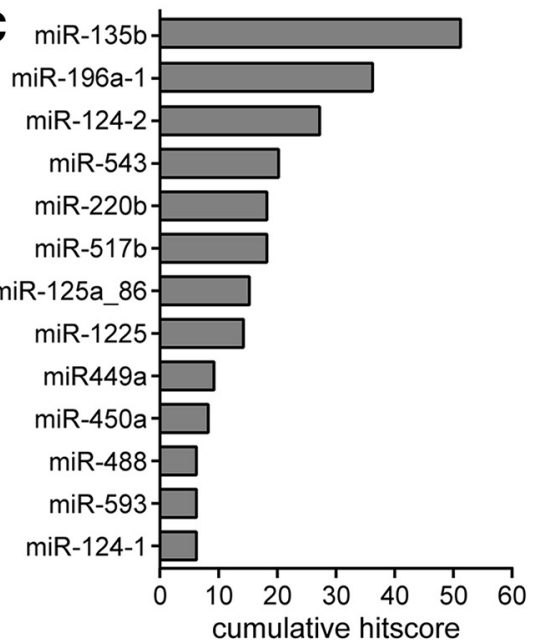

\begin{tabular}{|l|l|}
\hline \multirow{2}{*}{ miR-135b } & UAUGGCUUUUCAUUUCCUAUGUGA \\
\cline { 2 - 3 } & chr1: 205448302-205448398 \\
\hline miR-135a & UAUGGCUUUUㄴUUCCUAUGUGA \\
\cline { 2 - 2 } miR-135a-1 & chr3: 52294219-52294308 \\
\cline { 2 - 2 } miR-135a-2 & chr12: $97563812-97563911$ \\
\hline
\end{tabular}

E

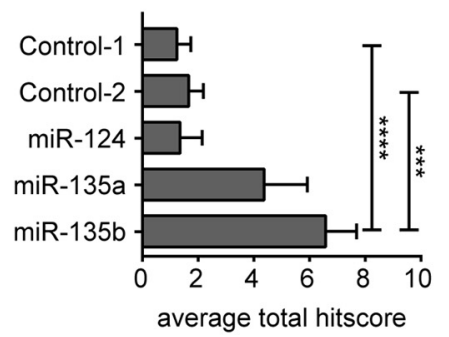

SH-SY5Y cells

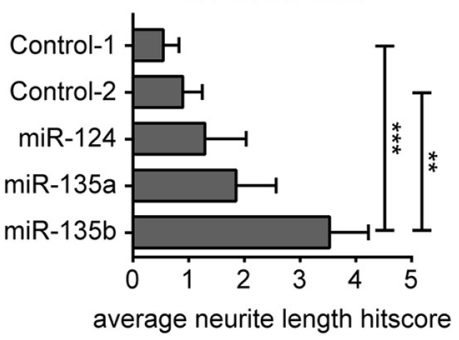

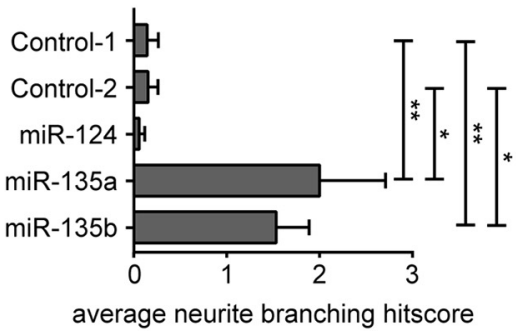

Figure 1. Image-based high-content screen identifies miRNAs involved in neurite growth. $\boldsymbol{A}$, Schematic representation of the Cellomics ArrayScan screen. SH-SY5Y cells were seeded and differentiated using retinoic acid. Viral library was added and after $3 \mathrm{~d}$ cells were fixed and immunostained. Images covering the entire surface of the well were taken using a Thermo Fisher Arrayscan automated microscope and analyzed using a neuronal profiling algorithm to assess the effect of miRNAs on general neuron-like features, such as the number of neurites, neurite length, and number of branch points. The effect of a miRNA on each parameter was scored binarily ( 0 or 1 ). A positive score (1) was given when the effect on the parameter deviated $>2$ times the SD of the median value for all miRNAs. Scores for each of the triplicate plates were combined, with the score for a certain parameter taken into account (effect is "true"') when the miRNA scored positive in a minimum of two of three plates. This resulted in a final (cumulative) "hitscore," which was used to rank the lentiviral clones for an effect on neuronal morphology. $\boldsymbol{B}$, Representative images of untreated SH-SY5Y cells (left) and SH-SY5Y cells treated with retinoic acid (middle). Right, Results of tracings generated by the neuronal profiling algorithm. Scale bar, $100 \mu \mathrm{m}$. C, Graph showing the cumulative score of all the parameters of the neuronal profiling algorithm for the top list of annotated miRNAs that have a positive effect on neuronal features of virus-transduced SH-SY5Y cells. $\boldsymbol{D}$, Sequence and genomic location of miR-135a and miR-135b. The mature sequence of miR-135a and miR-135b differs by only one nucleotide (underlined) outside of the seed region (in red). $\boldsymbol{E}$, Graphs showing average total hitscore (left), hitscore based on parameters describing neurite length (middle), and hitscore based on parameters describing neurite branching (right) of SH-SY5Y cells electroporated with the indicated miRIDIAN miRNA mimics. Data are expressed as means \pm SEM. ${ }^{*} p<0.05,{ }^{* *} p<0.01,{ }^{* *} p<0.001,{ }^{* * *} p<0.0001$, one-way ANOVA with Tukey's multiple-comparisons test. Scale bar, $200 \mu$ m. 
A

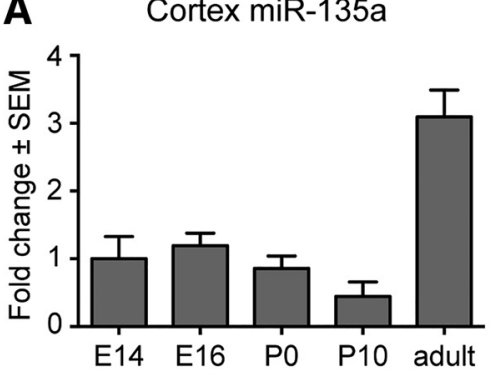

Cortex miR-135b

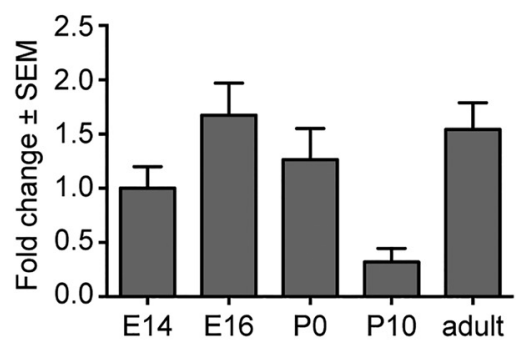

C Hippocampus miR-135a

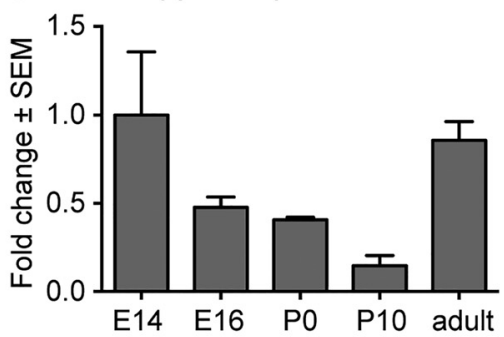

B
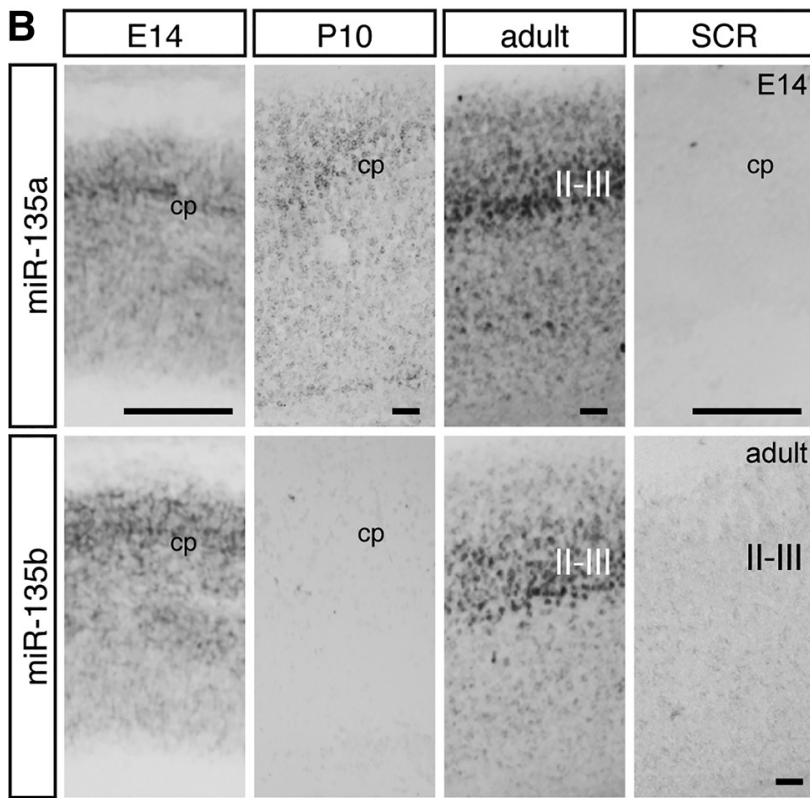

cp

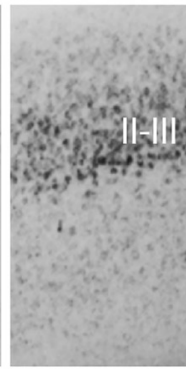

adult

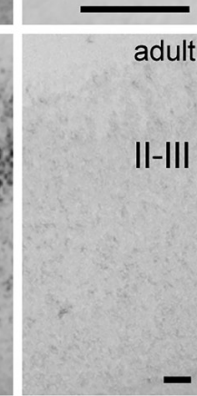

Hippocampus miR-135b

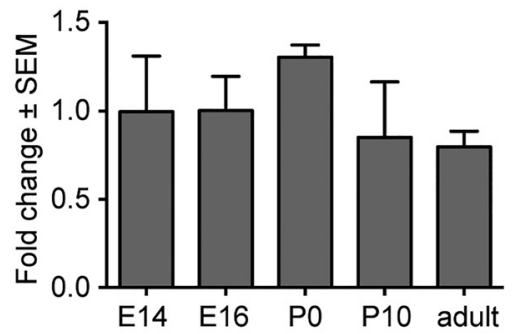

D
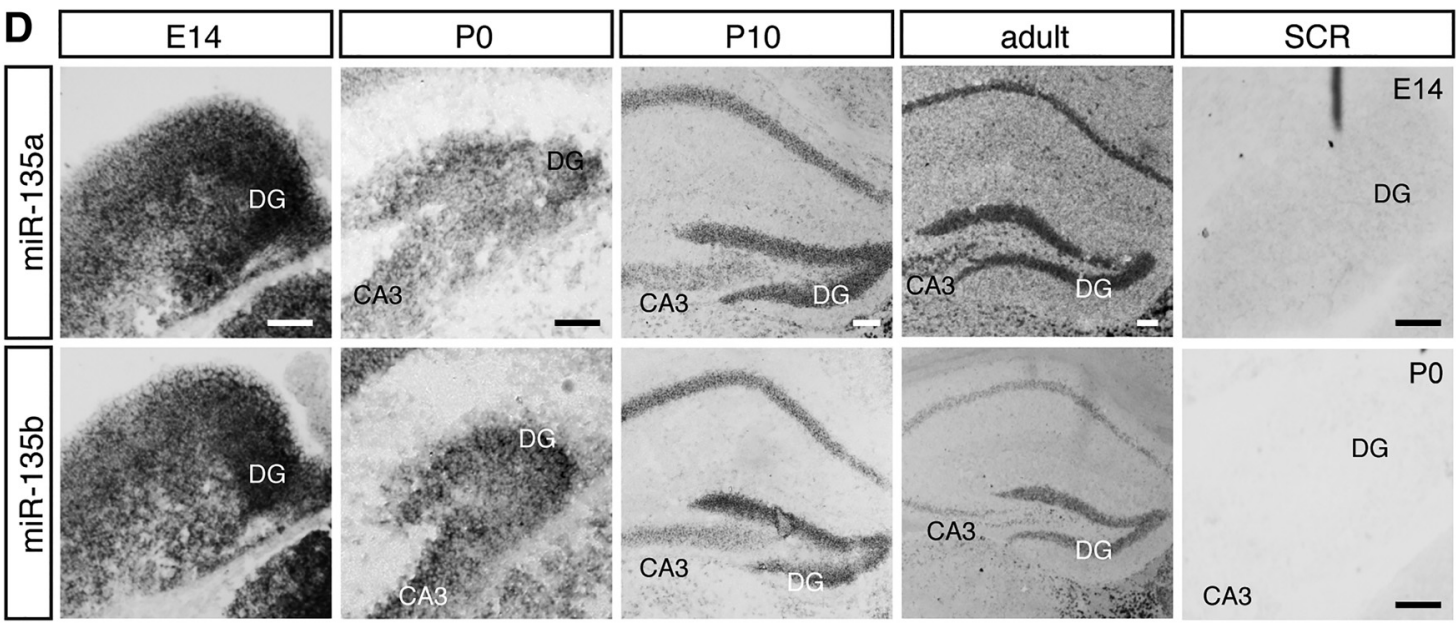

Figure 2. Expression of miR-135a and miR-135b during neuronal development. $\boldsymbol{A}, \boldsymbol{C}$, Graphs showing results of qPCR experiments on RNA from isolated mouse cortex $(\boldsymbol{A})$ or hippocampus $(\boldsymbol{C})$ from five different embryonic and postnatal stages. Tissue from three different mice from different litters was used for analysis. Samples were run in duplicate. Fold changes are relative to $5 \mathrm{~S}$ housekeeping rRNA expression. Data are expressed as means \pm SEM. B, LNA in situ hybridization shows miR-135a and miR-135b expression in the E14, P10, and adult cortex. miR-135a and miR-135b are expressed in the CP and upper layers of the adult cortex. Sections treated with scrambled LNA in situ probes were devoid of specific staining. Scale bars, $200 \mu \mathrm{m}$. D, LNA in situ hybridization showing miR-135a and miR-135b expression in the E16, P0, P10, and adult hippocampus. In the hippocampus, the dentate gyrus (DG) and CA3 region specifically show strong miR-135a and miR-135b staining. Sections treated with scrambled LNA in situ probes were devoid of specific staining. Scale bars, $200 \mu \mathrm{m}$.

transfections were replicated at least 3 times in triplo (i.e., $3 \times 3$ coverslips). miRNA mimics were cotransfected with GFP vector and, in the case of sponge vectors, internal RFP was exploited to trace neurite length using the NeuronJ plugin of ImageJ (Fig. $3 B-F$ ). Student's $t$ tests were performed to compare the means of each group with the control condition.
To assess the endogenous effects of miR-135a and miR-135b during neuronal development, ex vivo electroporation of miRNA mimics combined with a GFP vector and subsequent organotypic slice cultures of mouse embryonic cortex were performed at E14 (Fig. 4A). Embryos of one mother were divided among the three conditions to compare litter- 
A
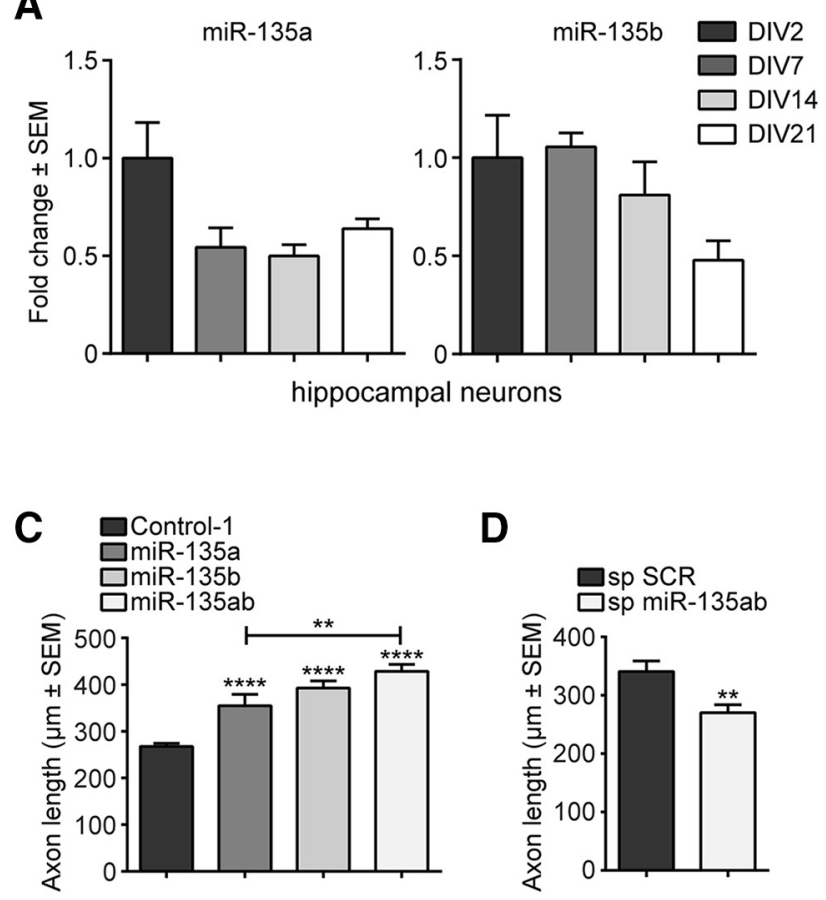

D

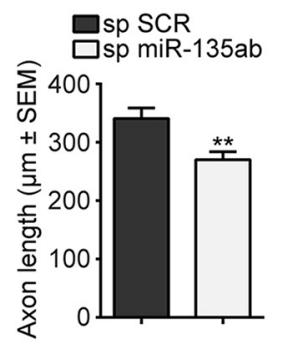

B

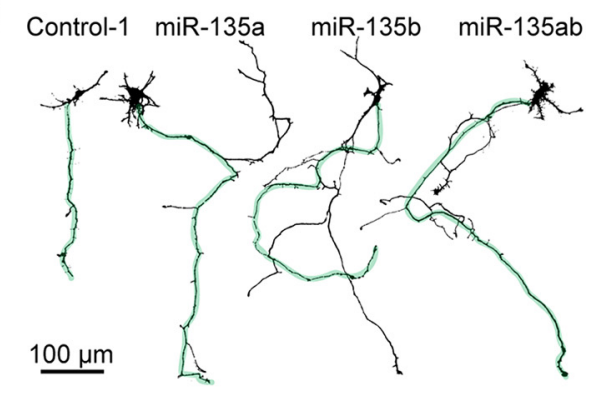

E

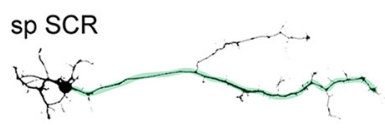

sp miR-135ab

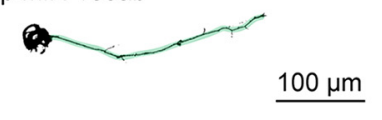

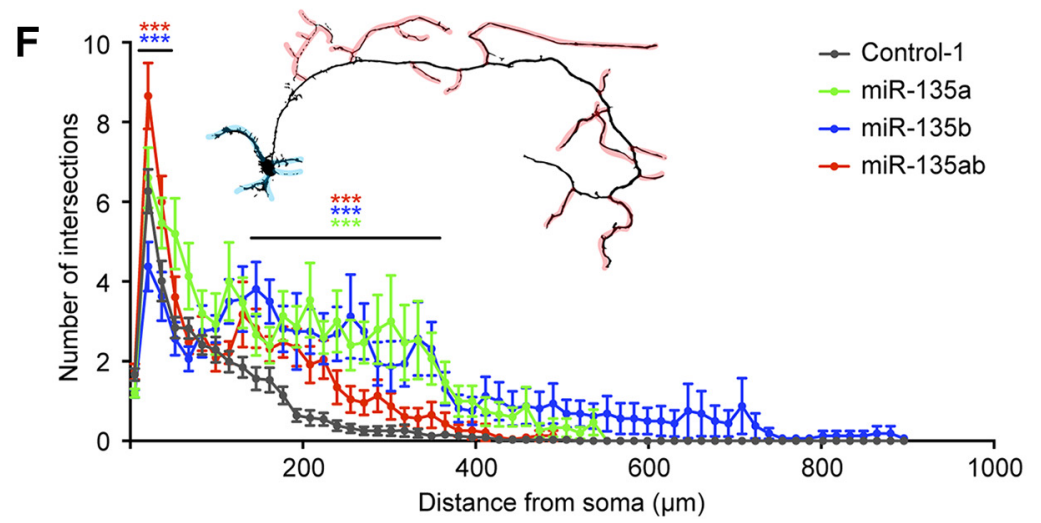

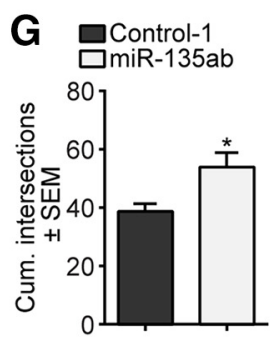

Figure 3. miR-135a and miR-135b increase neurite outgrowth and branching. $A$, Graphs showing results of qPCR on primary hippocampal neurons at different DIV. RNA was collected from three to four coverslips of two different cultures. Samples were run in duplicate. Fold changes are relative to $5 S$ housekeeping rRNA expression. Data are expressed as means \pm SEM. $\boldsymbol{B}$, Representative silhouettes of primary neurons at DIV4 after transfection with control-1, miR-135a, miR-135b, and miR-135a/miR-135b mimics. Longest neurites are labeled in green. $C$, Graph shows results of tracing of the longest neurite of DIV4 hippocampal neurons in experiments as in $A$. At least 173 neurons were traced from $\geq 3$ individual experiments. Data are expressed as means \pm SEM. ${ }^{* *} p<$ $0.01,{ }^{* * * *} p<0.0001, t$ test. D, Quantification of tracing of the longest neurite of DIV4 hippocampal neurons after transfection with scrambled or miR-135a and miR-135b H1-mCherry-sponge vectors. At least 100 neurons were traced from $\geq 3$ individual experiments. Data are expressed as means $\pm S E M .{ }^{* *} p<0.01, t$ test. $\boldsymbol{E}$, Representative silhouettes of primary neurons at DIV4 after transfection with control sponge- or miR-135ab sponge vector. Longest neurites are labeled in green. $\boldsymbol{F}$, Sholl analysis from 31 control-1 (gray), 15 miR-135a (green), 16 miR-135b (blue), or 23 miR-135a and miR-135b (red)-overexpressing neurons reveals increased branching in proximal neurites and in the distal axon. Data are expressed as means \pm SEM. ${ }^{* * * *} p<0.0001$, multiple $t$ tests. In the silhouette, proximal neurites are marked in blue and distal branches are marked in red. G, Cumulative intersections of neurites from neurons transfected with control-1 or miR-135a and miR-135b mimics with the Sholl circles (as in D). Data are expressed as means \pm SEM. ${ }^{* *} p<0.01, t$ test.

mates and the experiment was repeated three times. Similar cortical slices of at least six embryos from three different mothers were used for comparison. Next, in utero electroporation experiments were performed in E14 mice embryos to overexpress and downregulate miR-135a and miR$135 \mathrm{~b}$ in vivo. For embryonic analysis, the three conditions were divided over the embryos that were present in the uterus to always compare littermates. In utero electroporation dedicated to the isolation of postnatal tissue was performed in one condition per mother. For analysis of migration and neurite outgrowth of electroporated cortical neurons, three consecutive cryosections showing the corpus callosum were used and taken from at least nine pups derived from at least three different mothers. For both ex vivo and in utero electroporation analyses, MannWhitney $U$ tests were used to compare the distribution of the migrating cells. These were counted manually in two to three rasters containing eight cortical "bins" per slice placed exactly perpendicular to the direction of migration, with the bottom of bin 1 touching the border of the ventricle (for embryonic brains) or the axons of the anterior commissure (for postnatal brains) and the top of bin 8 reaching the cortical surface (Fig. 4A).

Next, possible mRNA targets of miR-135a and miR-135b were identified using the bio-informatic tool miRecords (Xiao et al., 2009). KLF4 was selected based on its reported effects on neurite outgrowth and neuronal migration. The strongest predicted binding site of miR-135a and miR-135b in the KLF4 3'-UTR was selected and used for a luciferase assay performed three times in HEK293 cells to confirm direct target binding (Fig. $5 A, B$ ). Immunohistochemistry was then used to assess 

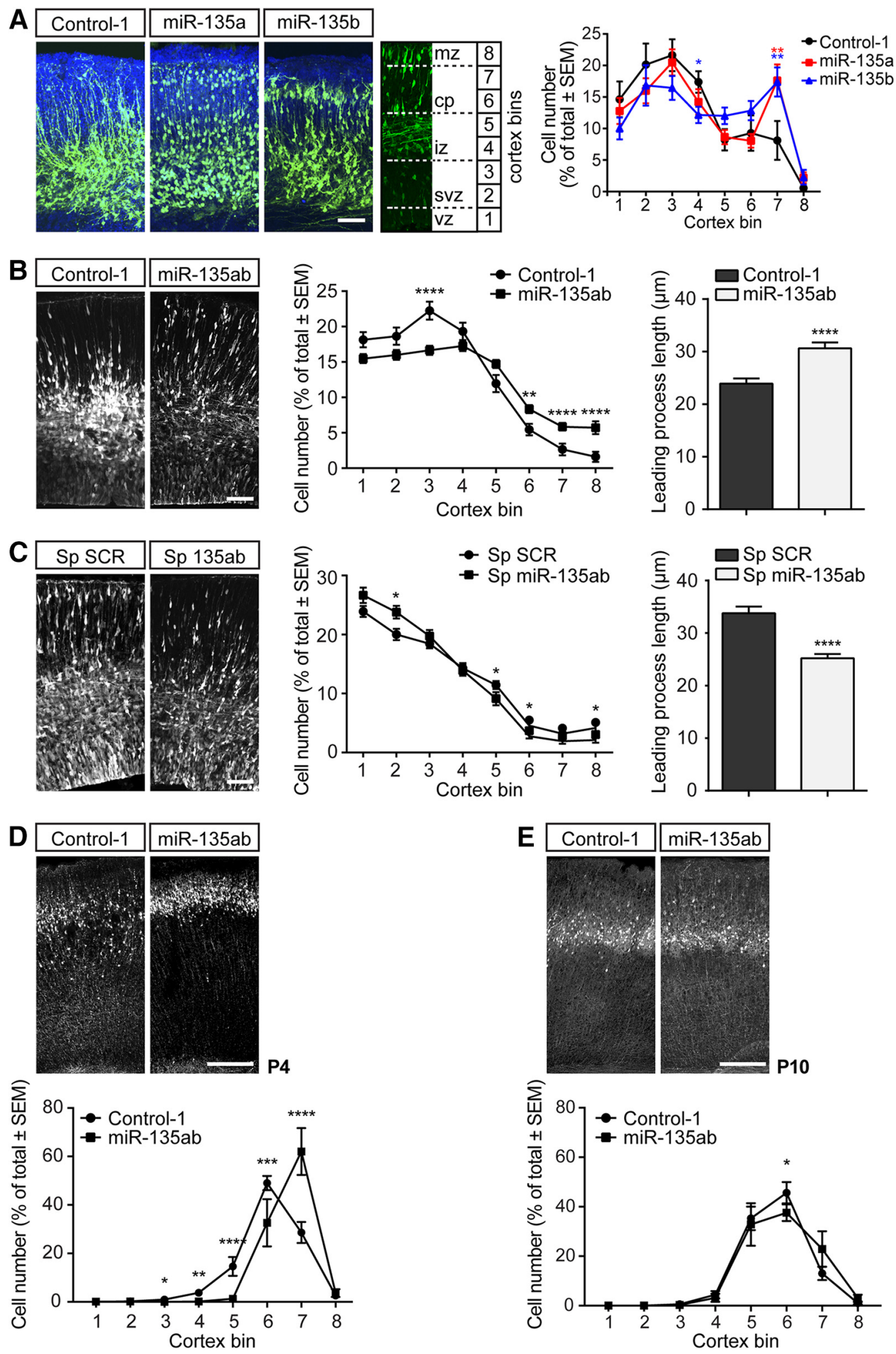

Figure 4. miR-135s are required for cortical neuron migration. A, Representative images of cortices that were ex vivo electroporated with control-1, miR-135a, or miR-135b mimics. Neuron migration was quantified by placing a rectangle containing eight square bins perpendicular on the cortex. Cells in each bin were counted and expressed as percentage of the total number of cells in the rectangle. The bins perfectly align with the layers of the cortex: VZ, subventricular zone (SVZ), intermediate zone (IZ), CP, and marginal zone (MZ). Cell counts of two to three rectangles per section were used for comparison. At least two to three sections from $\geq 3$ animals from different litters were used. Data are expressed as means \pm SEM. Red ${ }^{* *}$ bin7 control- 1 vs miR-135a: Mann-Whitney $U$ test $(M W U)=24, p=0.0042$; blue ${ }^{*}$ bin 4 control-1 vs miR-135b: MWU $=32, p=0.0195$; blue ${ }^{* *}$ bin 7 control-1 vs miR-135b: MWU $=25, p=0.0051$, tests. Scale bar, $100 \mu$ m. $B$, Representative images and quantification of neuron migration and leading process length in in utero electroporated E16.5 cortices of mice embryos treated with either control-1 or miR-135a and miR-135b mimics. GFP signal is shown in white. Neuron migration was quantified as described in $F$. Data are expressed as means \pm SEM. Bin 3: MWU $=198, p<0.0001$; bin 6 MWU $=282, p<$ 0.0053; bin 7: MWU $=161, p<0.0001$; bin 8: MWU $=164, p<0.0001 .{ }^{* *} p<0.01,{ }^{* * *} p<0.0001$, MWU; ${ }^{* * * *} p<0.000,1 t$ test. Scale bar, $100 \mu \mathrm{m}$. C, Representative images and quantification of neuron migration and leading process length in in utero electroporated E16.5 cortices of mice embryos treated with either scrambled or miR-135a and miR-135b H1-mCherrysponge vectors. $\mathrm{mCherry} \mathrm{signal} \mathrm{is} \mathrm{shown} \mathrm{in} \mathrm{white.} \mathrm{Neuron} \mathrm{migration} \mathrm{was} \mathrm{quantified} \mathrm{as} \mathrm{described} \mathrm{in} \boldsymbol{A}$. Data are expressed as means \pm SEM. Bin 2: MWU $=70$, (Figure legend continues.) 
whether KLF4 and miR-135a and miR-135b are expressed in similar brain areas (Fig. 5C). Next, we tested whether endogenous KLF4 expression in N2A cells was downregulated upon miR-135a and miR-135b administration. To determine an endogenous role for miR-135-KLF4 signaling, rescue experiments were performed in primary hippocampal neuron cultures and in utero electroporation using KLF4 cDNA (insensitive to miRNA regulation) using the same experimental procedures and repetitions as described before in the in utero experiments described in Fig. 4.

Because KLF4 is one of the most important signals counteracting axon regeneration, we investigated whether miR-135a and miR-135b could be used to decrease KLF4 expression in poorly regenerating neurons in a specific and cell-autonomous fashion. We first injected miRNA mimics intravitreally (on day 0 and day 7) to learn whether this was sufficient to deliver miRNAs to the optic nerve and to downregulate KLF4 expression. qPCR was performed on three optic nerves per condition $14 \mathrm{~d}$ after the first injection of mimics. Then, mimics were combined with GFP vector and/or KLF4 cDNA to determine axon regeneration $14 \mathrm{~d}$ after ONI. This was repeated in nine mice per condition. qPCR experiments revealed no differences in transfection efficiency between conditions. AAV2 virus containing miR-135a, miR-135b, or control miRNA was injected to transduce RGCs only in 6 mice $7 \mathrm{~d}$ before the optic nerve crush and to assess the cell-autonomous nature of the effect observed with mimic injections. Finally, we determined whether miR-135a and miR-135b had an endogenous role in optic nerve regeneration measured $14 \mathrm{~d}$ after the optic nerve crush by injecting sponge vectors at day 0 and day 7 in 6 mice. Axon regeneration was tested statistically by ANOVAs followed by Sidak post hoc tests.

\section{Results}

\section{miRNome-wide screen for miRNAs that regulate neurite growth}

To identify miRNAs that can promote neurite growth, an imagebased miRNA screen was performed in neuronal SH-SY5Y cells, a cell line regularly used for cellular screening. Neuronal differentiation of SH-SY5Y cells was induced by retinoic acid treatment followed by transduction of a lentiviral library containing 1140 unique human miRNAs (Poell et al., 2011; Fig. 1A,B). Using a Cellomics ArrayScan platform, thousands of cells in each condition were analyzed for parameters related to neuronal morphology. This multiparametric analysis resulted in a cumulative hitscore that was based on parameters such as neurite length and branching (Table 1). To identify hits, scores for each individual miRNA were compared with the median score of all miRNAs. This approach assumes that the majority of miRNAs do not affect neuronal morphology. This approach identified 13 annotated miRNAs with pronounced effects on specific morphological properties of differentiated SH-SY5Y cells (e.g., neurite length). Of these miRNAs, miR-135b had the largest effect (Fig. 1C).

To confirm the effect of miR-135b, retinoic acid-treated SHSY5Y cells were electroporated with miR-135b mimics to simu-

\section{$\leftarrow$}

(Figure legend continued.) $p=0.018$; bin 5: MWU $=69, p=0.016$; bin $6:$ MWU $=75, p=$ 0.030 ; bin 8: MWU $=69, p=0.016, \mathrm{MWU}^{*} p<0.05 ;{ }^{* * * *} p<0.0001, t$ test. Scale bar, 100 $\mu \mathrm{m} . \boldsymbol{D}$, Representative images and quantification of neuron migration in in utero electroporated P4 cortices of mice pups electroporated with either control- 1 or miR-135a and miR-135b mimics at E14.5. GFP signal is shownin white. Neuron migration was quantified as described in A. Data are expressed as means \pm SEM. Bin3: $\mathrm{MWU}=475, p=0.0114$; bin 4: $\mathrm{MWU}=392.5$, $p=0.0016$; bin $5: M W U=148, p<0.0001$; in $6: M W U=319.5, p=0.0004$; bin 7: $\mathrm{MWU}=$ 194.5, $p<0.0001$, MWU. ${ }^{*} p<0.05,{ }^{* *} p<0.01,{ }^{* * *} p<0.001,{ }^{* * * *} p<0.0001$. Scale bar, $200 \mu \mathrm{m}$. $\boldsymbol{E}$, Representative images and quantification of neuron migration in in utero electroporated P10 cortices of mice pups electroporated with either control-1 or miR-135a and miR$135 \mathrm{~b}$ mimics at E14.5. GFP signal is shown in white. Neuron migration was quantified as described in $A$. Data are expressed as means \pm SEM. Bin 6: MWU 783.5, $p=0.032$, MWU. ${ }^{*} p<$ 0.05. Scale bar, $200 \mu \mathrm{m}$. late overexpression. miR-135a, a close homolog of miR-135b (Fig. 1D), was also included because it shares many mRNA targets with miR-135b and because we suspected that miR-135a was not identified in the initial screen because of technical problems (edge-well effects in the culture plates). miR-124, a well known brain-enriched miRNA that was identified in the screen, was also included (Fig. 1C), as well as two control miRNA mimics (both originating from $C$. elegans and proven to not target specific mammalian mRNAs (Dharmacon) (personal observations). Consistent with the results of the screen, miR-135b mimics affected the general morphology of SH-SY5Y cells $[6.58 \pm 1.11$ vs $1.24 \pm 0.50, t_{(188)}=4.64, p<0.0001$ (control-1), or vs $1.67 \pm$ $0.52, t_{(188)}=4.19, p<0.0001$ (control-2), one-way ANOVA, Sidak post hoc test; Fig. 1E, left]. Furthermore, miR-135b enhanced neurite outgrowth $\left[3.53 \pm 0.69, t_{(189)}=4.46, p<0.0001\right.$ vs $0.55 \pm 0.28$ (control- 1 ) or vs $0.90 \pm 0.35 t_{(189)}=3.87, p=$ 0.0006 (control-2), one-way ANOVAs, Sidak post hoc tests; Fig. $1 E$, middle]. Total hitscore and hitscore related to neurite length appeared to be affected by miR-135a, but these effects did not reach statistical significance. Neurite branching was significantly increased by miR-135a $\left(2.00 \pm 0.71, t_{(189)}=3.62, p=0.0015 \mathrm{vs}\right.$ control- 1 and $t_{(189)}=3.54 ; p=0.0020$ vs control-2) and miR$135 \mathrm{~b}\left(1.53 \pm 0.36, t_{(189)}=3.11, p=0.0085\right.$ vs control- 1 and $t_{(189)}=$ 3.03, $p=0.011$ vs control-2, one-way ANOVAs, Sidak post hoc tests) mimics compared with control mimics $[0.14 \pm 0.12$ (control-1), or $0.15 \pm 0.11$ (control-2); Fig. $1 E$, right]. Together, these data confirm that miR-135b and miR-135a increase neurite growth and complexity.

\section{Expression of miR-135a and miR-135b in the developing mouse cortex and hippocampus}

miR-135a and miR-135b sequences are preserved across species and detected in mouse brain tissue (Lagos-Quintana et al., 2002; Sempere et al., 2004; Ziats and Rennert, 2014; Caronia-Brown et al., 2016). However, the precise spatiotemporal pattern of expression and functional role of these miRNAs in neurons remained poorly understood. Therefore, we first analyzed the expression of miR-135a and miR-135b by qPCR in the developing (at E14, E16, $\mathrm{P} 0$, and $\mathrm{P} 10$, during which neurite growth and branching occur) and in adult mouse cortex and hippocampus. qPCR analysis detected miR-135a and mR-135b in embryonic, postnatal, and adult cortex and hippocampus. Expression of both miRNAs declined as cortical development progressed, but was increased again at adult stages. In contrast, whereas hippocampal miR-135a expression decreased toward P10 and displayed higher levels again at adult stages, miR-135b levels remained unchanged (Fig. $2 A, C)$. LNA-based in situ hybridization was used to reveal the spatial distribution of both miRNAs and revealed miR-135a and miR-135b expression in the cortex (at E14, P10, and adult; Fig. $2 B$ ) and hippocampus (at E14, P0, P10, and adult; Fig. 2D). Specific signals were observed in the dentate gyrus and CA3 pyramidal cell layers of the hippocampus and in the cortical plate (CP) of the developing cortex. Furthermore, both miRNAs were expressed in the adult mouse brain (Fig. $2 B, D$ ). Therefore, miR$135 \mathrm{a}$ and miR-135b display specific spatiotemporal patterns of expression in the developing mouse brain.

miR-135a and miR-135b control axon growth and branching miR-135a and miR-135b displayed prominent hippocampal expression; therefore, to investigate their functional role in neurons, hippocampal neurons were dissociated, transfected with miRNA mimics, and analyzed for axon growth at DIV4. First, qPCR was used to confirm endogenous expression of miR-135a 


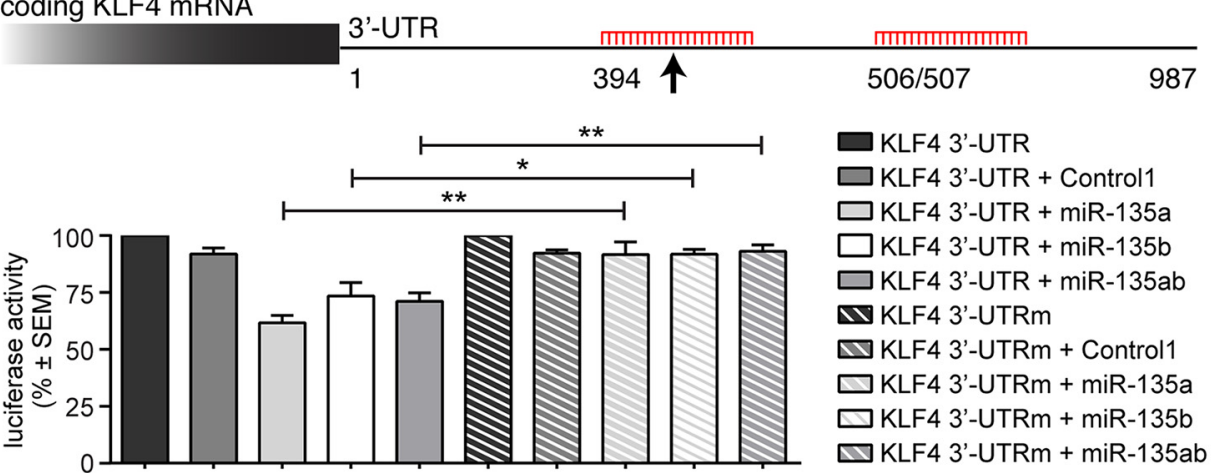
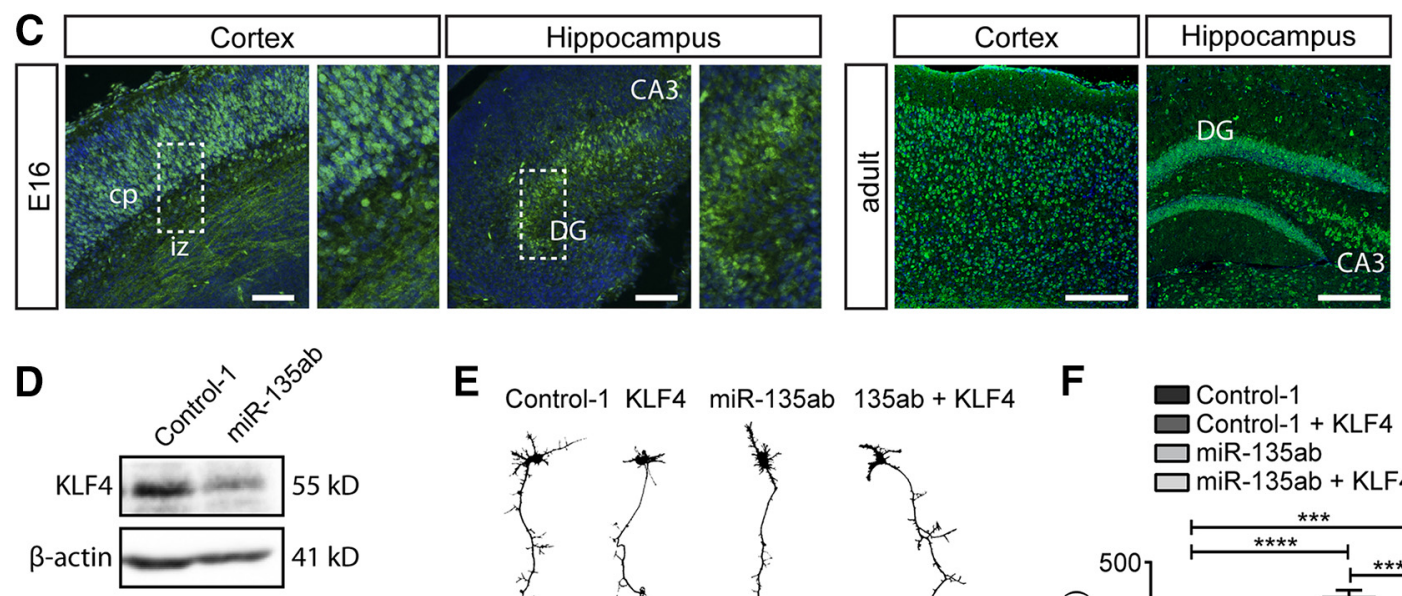

E

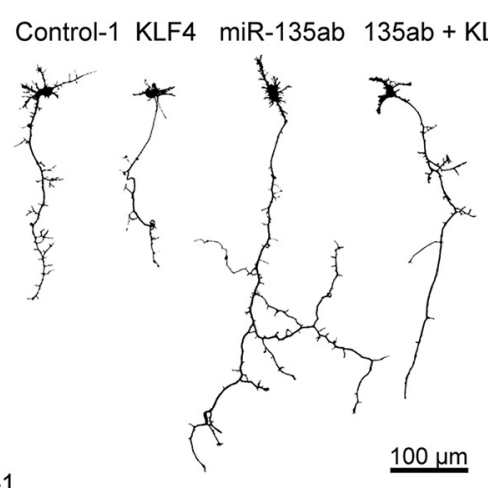

$\mathbf{F}$
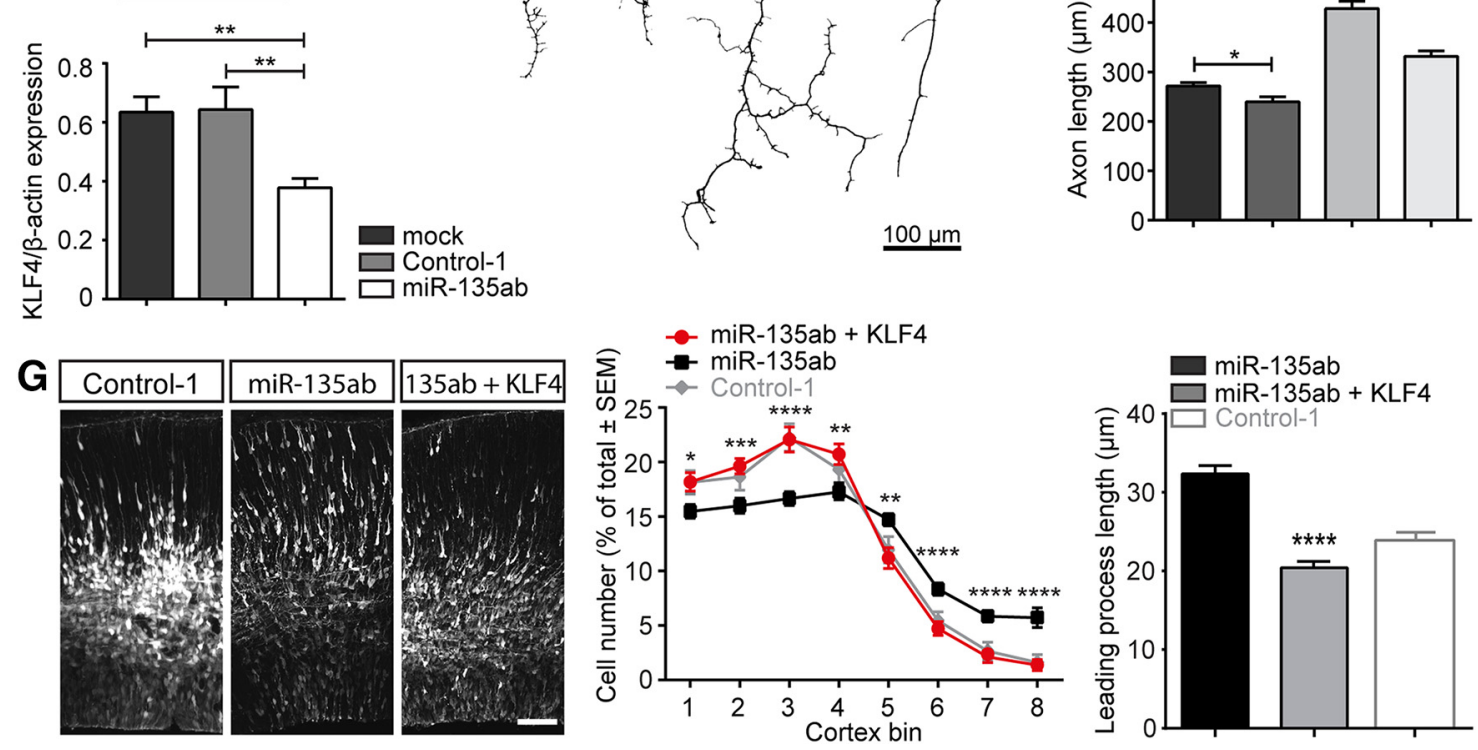

Figure 5. KLF4 is a functional target for miR-135a and miR-135b during axonal development and neuron migration. $A$, Schematic representation of predicted miR-135a and miR-135b-binding sites in the 3'-UTR of KLF4 mRNA. Site 394 is predicted to mediate strongest binding (arrow). B, 3'-UTR of KLF4 was cloned into a psi-CHECK2 vector and used for a Renilla-luciferase assay with control-1 or miR-135a and miR-135b mimics. Subsequently, a psi-CHECK vector with the KLF43'-UTR in which $3 \mathrm{nt}$ within site 394 were mutated was used to confirm specificity of miRNA-135-KLF4 binding. Luciferase activity was normalized to the 3'-UTR only condition of either wild-type or mutated $3^{\prime}$-UTR (UTRm). The experiment was repeated three times. Data are expressed as means \pm SEM. ${ }^{* *} p<0.01,{ }^{*} p<0.05, t$ test. C, Immunohistochemistry of KLF4 in sections of mouse cortex and hippocampus at E16.5 and adulthood. KLF4 is highly expressed in the CP, in axons running through the intermediate zone (IZ), in hippocampal granule cells in the dentate gyrus (DG), and in pyramidal cells of the CA3. Scale bars, $200 \mu \mathrm{m}$. D, Western blot analysis of KLF4 protein levels after transfection of control- 1 or miR-135a and miR-135b mimics in Neuro2A cells. Data are expressed as means \pm SEM. ${ }^{* *} p<0.01, t$ test. $E$, Representative silhouettes of primary hippocampal neurons at DIV4 after transfection with control-1 mimics, control-1 mimics combined with a KLF4 CDNA insensitive for miRNA binding (CMV-KLF4-GFP), miR-135a and miR-135b mimics, and miR-135a and miR-135b mimics combined with CMV-KLF4-GFP.F, Graph showing the results of tracing of the longest neurite of DIV4 hippocampal neurons in experiments as in $E$. At least 182 neurons were traced from $\geq 3$ individual experiments. Data are expressed as means \pm SEM. ${ }^{*} p<0.05,{ }^{* * *} p<0.001,{ }^{* * * *} p<0.0001, t$ test. G, Representative images and quantification of neuron migration and leading process length in in utero electroporated E16.5 cortices of mice embryos treated with either miR-135a and miR-135b mimics or miR-135a and miR-135b mimics combined with a pCAG-KLF4 vector insensitive to miR-135 regulation. Neuron migration was quantified as described in Figure 4. Data are expressed as means \pm SEM. Bin 1: Mann-Whitney $U$ test (MWU) $=422, p=0.0171$, $\operatorname{bin} 2: \mathrm{MWU}=332, p=0.0005$, in 3: $\mathrm{MWU}=293, p<0.0001$, bin 4: $\mathrm{MWU}=395, p=0.0068$, bin 5: $\mathrm{MWU}=357, p=0.0016$, bin $6: \mathrm{MWU}=261, p<0.0001$, bin 7:MWU $=219, p<0.0001$ and bin 8: MWU $=211.5, p<0.0001, \mathrm{MWU} .{ }^{*} p<0.05,{ }^{* *} p<0.01,{ }^{* *} p<0.001,{ }^{* * *} p<0.0001$. Control-1 condition is as described in Figure $4 B$. Scale bar, $100 \mu \mathrm{m}$. 
and miR-135b in primary hippocampal cultures (Fig. 3A). At DIV4, the longest neurite, confirmed to be the axon, was significantly longer in neurons transfected with miR-135a (354.9 \pm $24.41 \mu \mathrm{m})$ or miR-135b $(392.8 \pm 15.24 \mu \mathrm{m})$ mimics compared with control $\left[271.7 \pm 7.18 \mu \mathrm{m}, t_{(776)}=4.443\right.$ (control-1 vs miR$135 \mathrm{a}), t_{(900)}=8.181$ (control-1 vs miR-135b), both $p<0.0001$, unpaired $t$ tests; Fig. $3 B, C$ ]. Cotransfection of both miR-135a and miR-135b further increased axon length $(428.7 \pm 14.97 \mu \mathrm{m}$, vs control-1 $t_{(1022)}=10.36, p<0.0001$; vs miR-135a $t_{(590)}=$ 2.628, $p=0.0088$, unpaired $t$ tests). To assess the endogenous roles of miR-135a- and miR-135b, specific miRNA sponges designed to sequester miR-135a and miR-135b were cotransfected into hippocampal neurons. Decreased availability of the miRNAs resulted in a significant decrease in axon length $(270.1 \pm 13.63$ $\mu \mathrm{m})$ compared with scrambled control sponge transfection $\left(340.1 \pm 18.09 \mu \mathrm{m}, t_{(211)}=3.053, p=0.0026\right.$, unpaired $t$ test; Fig. $3 E$ ). Because the initial screen in SH-SY5Y cells showed effects on both neurite growth and branching, Sholl analysis was performed on primary hippocampal neurons transfected with miR-135a, miR-135b, and the combination of the two mimics. Overexpression of both miRNAs alone and combined resulted in a marked increase in neurite branching in more distal regions (Fig. 3F). Interestingly, combined overexpression of miR-135a and $\mathrm{miR}-135 \mathrm{~b}$ or of miR-135b alone also resulted in increased branching in the area close to the cell body. These data suggest an increase in the number of (branches of) primary neurites (blue neurites) and increased branching of the axon (red neurites; control-1 vs miR-135a: $t_{(12800)}$ ranges from 3.728 to 8.52 , control-1 vs miR-135b: $t_{(13144)}$ ranges from 3.735 to 6.426 ; control-1 vs miR-135ab: $t_{(12164)}$ ranges from 3.84 to $7.496 ; p<0.001$ for all; unpaired $t$ tests). The number of cumulative intersections of neurites with the Sholl circles was also higher in miR-135ab treated neurons $(53.9 \pm 4.91)$ compared with control $\left(38.69 \pm 2.67, t_{(37)}=2.414, p=0.021\right.$, unpaired $t$ test; Fig. $3 G$ ). Together, these experiments show that miR-135a and miR-135b regulate axon growth and branching.

Cortical neuron migration requires miR-135a and miR-135b miR-135a and miR-135b are expressed in hippocampal and cortical neurons as they migrate in the developing nervous system and extend neurites (Fig. 2) and manipulation of these miRNAs affects neuronal morphology in cultured hippocampal as well as cortical neurons (Fig. 3 and data not shown). To assess the role of miR-135a and miR-135b in neurons in the complex environment of the embryonic brain, we performed ex vivo and in utero electroporation (van Erp et al., 2015). Ex vivo electroporation of mouse cortex with miR-135a and miR-135b mimics was performed at E14.5 and brains were sliced and cultured and analyzed at DIV4. Electroporation of miR-135a or miR-135b mimics induced a marked increase in the migration of cortical neurons from the VZ to the CP, exemplified by a larger number of electroporated GFP-positive neurons in the CP and fewer cells in the intermediate zone compared with control mimic conditions (Fig. $4 A$, see figure legend for statistical results). To confirm these effects in vivo, we delivered miRNA mimics or sponges to the E14.5 cortex by in utero electroporation and analyzed migrating neurons at E16.5. Mimics for miR-135a and miR-135b were combined to elicit significant phenotypes in a short time period. Consistent with the ex vivo electroporation data, delivery of miR$135 \mathrm{ab}$ mimics to the cortex enhanced neuronal migration toward the pial surface and induced a concomitant depletion in deeper layers such as the subventricular zone (Fig. 4B, see figure legend for statistical results). Electroporation of miR-135a and miR$135 \mathrm{~b}$ sponges had a small, but opposite effect, delayed migration of cortical neurons, confirming an endogenous requirement for miR-135a and miR-135b in cortical neuron migration (Fig. 4C, see figure legend for statistical results). As a measure for in vivo neurite outgrowth, we quantified the length of the leading process of migrating neurons after in utero electroporation. Although miR-135a and miR-135b mimics induced an increase in leading process length $\left(30.65 \pm 1.09\right.$ vs $23.91 \pm 1.01, t_{(364)}=$ 4.497, $p<0.0001$, unpaired $t$ test; Fig. $4 B$ ), leading processes were shorter after application of miR-135 sponges $(25.23 \pm 0.80$ vs $33.78 \pm 1.28, t_{(325)}=5.712, p<0.0001$, unpaired $t$ test; Fig. $4 C$ ). To assess the long-term effect of miR-135 overexpression in vivo, we isolated the brains from embryos in utero electroporated at E14.5 at P4 (Fig. 4D) and P10 (Fig. 4E). Interestingly, at P4 electroporation of miR-135a and miR-135b enhanced neuron migration significantly, resulting in a larger number of neurons in upper cortical areas (Fig. 4D, see figure legend for statistical results). At P10, a small but significant difference in the distribution of cells in the upper cortical layers remained between embryos electroporated with control or miR135 mimics (Fig. 4E, see figure legend for statistical results). Overall, these data suggest that, consistent with their effects in cultured neurons, miR-135a and miR$135 \mathrm{~b}$ control neurite length and neuron migration in vivo.

\section{miR-135a and miR-135b control axon growth and neuronal migration through KLF4}

How do miR-135a and miR-135b control neuronal morphology and migration? Based on high sequence similarity and comparable biological effects in neurons, we hypothesized that miRNA135a and miRNA-135b may share many of their mRNA targets. To identify those targets, we performed target prediction analysis using miRecords (Xiao et al., 2009). By combining data from at least six databases in miRecords, 57 overlapping targets were found for miR-135a and miR-135b (Table 2). Several of these targets had confirmed roles in neurite growth and neuronal morphology. However, for many of these targets (e.g., PTK2, TAF4), knock-down had been reported to reduce neurite growth or neuron migration (data not shown). KLF4 was particularly interesting because knock-down of KLF4 in neurons, similar to overexpression of miR-135s, enhances axon growth, leading process length, and neuronal migration (Moore et al., 2009; Qin and Zhang, 2012). Furthermore, recent work in vascular smooth muscle and hepatocellular carcinoma cells links miRNA-135a to KLF4 (Lin et al., 2016; Yao et al., 2016). Finally, KLF4 contains predicted binding sites for several miRNAs in the top list of our initial screen (miR-124, miR-449, miR-488, miR-499; Fig. 1C). The 3'-UTR of KLF4 harbors two predicted miR-135-binding sites (Fig. 5A) and, to confirm that KLF4 is a bona fide target for miR-135s, we first performed dual-luciferase reporter assays by cotransfecting psiCHECK2-KLF4 3'-UTR and miR-135a and miR-135b mimics into HEK293 cells. miR-135a and miR-135b mimics decreased luciferase activity significantly both when transfected alone or when combined (Fig. 5B). To confirm direct and specific binding, the miRNA-135-binding site that was predicted to have the strongest association (according to www. microRNA.org) was mutated (site 394; Fig. 5A). This mutation completely abolished miR-135-mediated effects on luciferase activity, suggesting that site 394 is the main miR-135-binding site in KLF4 (KLF4 WT miR-135a vs KLF4 mutated miR-135a, $t_{(4)}=$ $4.715, p=0.0092$; KLF WT miR-135b vs KLF4 mutated miR$135 \mathrm{~b}, t_{(4)}=2.933, p=0.0427$; KLF4 WT miR-135ab vs KLF4 mutated miR-135ab, $t_{(4)}=4.735, p=0.0091$, unpaired $t$ test; Fig. $5 B)$. Next, we performed immunohistochemistry for KLF4 to assess whether miR-135s and KLF4 are expressed in the same 
Table 2. Potential shared mRNA targets for miR-135a and miR-135b

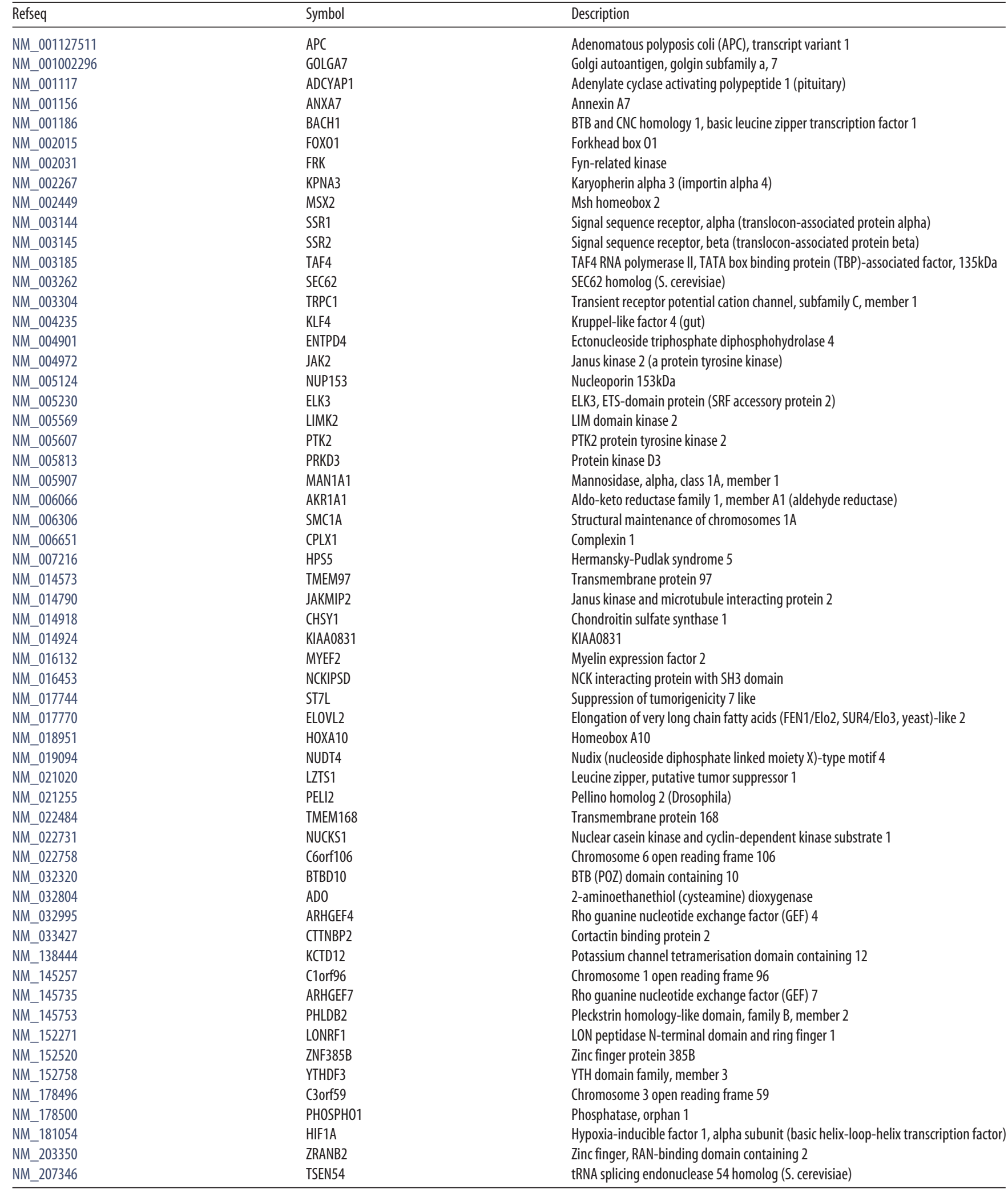

Predictions were made by the online bioinformatic tool miRecords. Within miRecords targets were predicted using at least six different prediction programs: MiRanda, MirTarget2, PicTar, PITA, RNAhybrid, and TargetScan/TargetScanS.

brain regions. Indeed, consistent with our in situ hybridization data for miR-135a and miR-135b, prominent KLF4 expression was detected in neurons in the CP of the E16.5 and adult cortex and in the developing and adult hippocampus (Fig. 5C). To further validate the relation between miR-135 and KLF4, endoge- nous KLF4 protein levels were analyzed in transfected Neuro2A cells by Western blot. Reduced KLF4 expression was observed after transfection with miR-135a and miR-135b mimics compared with control mimic transfection (miR-135ab vs control-1: $0.378 \pm 0.032$ vs $0.643 \pm 0.01, t_{(10)}=3.170, p=0.010$, unpaired 
$t$ test; Fig. $5 D$ ). Together, these data indicate that KLF4 is a target for miR-135a and miR-135b.

Next, we assessed whether the effects of miR-135s (miR-135a and miR-135b) on axon growth and neuronal migration require KLF4. In primary hippocampal neurons, cotransfection of KLF4 cDNA lacking the $3^{\prime}$-UTR (KLF4 $33^{\prime}$-UTR), and therefore miR135-binding sites, markedly reduced the increase in axon growth by transfection of miR-135 mimics (Fig. 5E, control-1 vs control- $1+$ KLF4: $271.1 \pm 7.178$ vs $239.9+9.701, t_{(785)}=2.250$, $p=0.025$; control-1 vs miR-135ab + KLF4: $271.1 \pm 7.178$ vs $331.7 \pm 10.92, t_{(980)}=4.787, p<0.0001 ;$ miR-135ab + KLF4 vs miR-135ab: $331.7 \pm 10.92$ vs $428.7 \pm 14.97, t_{(794)}=5.139, p<$ 0.0001 , unpaired $t$ test; Fig. $5 F$ ). Similarly, the positive effect of miR-135a and miR-135b overexpression on cortical neuron migration and leading process length was normalized by coelectroporation of KLF4 $\Delta 3^{\prime}$-UTR (Fig. $5 G$, see figure legend for statistical results of neuronal migration, for leading process length: miR-135ab: $32.34 \pm 1.084$, miR-135ab+KLF4: $20.42 \pm$ $0.79, t_{(240)}=8.851, p<0.0001$, unpaired $t$ test). Together, these experiments indicate that miRNA-135s enhance axon growth and neuronal migration by repressing KLF4 protein expression.

\section{Exogenous miR-135 application promotes optic nerve regeneration through KLF4}

Lowering neuronal KLF4 expression not only promotes axon growth in developing neurons but is also one of the few experimental treatments that facilitates regenerative axon growth after CNS injury. Knock-out mice lacking KLF4 showed significantly enhanced RGC axon regeneration after ONI (Moore et al., 2009; Qin et al., 2013) This effect of KLF4 requires downstream signaling via the Janus kinase (JAK)-signal transducer and activator of transcription 3 (STAT3) pathway (Qin et al., 2013), but upstream regulatory mechanisms of this pathway remain unknown. Because of these results and our data showing that miR-135 mimics can promote axon growth by reducing KLF4 expression, we next investigated whether application of miR-135 mimics can facilitate regenerative axon growth in the CNS. To test this hypothesis, we used the optic nerve crush model. siRNAs and miRNA mimics can be targeted efficiently to adult RGCs and optic nerve regeneration can be quantified reliably (Dickendesher et al., 2012; van Erp et al., 2015). Further, both KLF4 and miR-135s are expressed in adult mouse RGCs and reducing KLF4 expression enhances optic nerve regeneration (Moore et al., 2009; Qin et al., 2013). First, we confirmed that intravitreal injection of miR-135 mimics leads to an elevation of miR-135a and miR-135b levels (Fig. $6 A, B)$. Although endogenous expression of miR-135s was detected, intravitreal injection of mimics increased miR-135a and miR-135b expression markedly compared with injections with scrambled controls (control-1 vs miR-135a: $t_{(4)}=2.462, p=$ 0.0348; control-1 vs miR-135b: $t_{(4)}=4.309, p=0.0063$, unpaired $t$ test). Consistent with our data identifying KLF4 as a miR-135 target (Fig. 5), injection of miR-135 mimics in the eye led to a decrease in KLF4 expression (control-1 vs miR-135ab: $t_{(3)}=$ 2.901, $p=0.0312$, unpaired $t$ test; Fig. $6 C$ ). Next, we assessed the effect of miR-135 injection on optic nerve regeneration. After administration of scrambled control mimics, most CTB-labeled RGC axons stopped abruptly at the crush site and only few fibers crossed the lesion into the distal nerve (Fig. $6 D, E$ ). In contrast, miR-135 mimics induced significant regeneration $(0.2 \mathrm{~mm}$, control-1 + GFP vs miR-135ab + GFP: $46.89 \pm 6.816$ vs $208.4 \pm$ $35.11, t_{(96)}=7.374, p<0.0001$, one-way ANOVA with Sidak post hoc test) beyond the lesion site and more pronounced sprouting in the distal segment of the nerve (Fig. $6 D, E$ ). To determine whether this effect was caused by the ability of miR-135s to reduce KLF4 expression, we combined intravitreal injection of miR-135 mimics with cotransfection of vectors expressing GFP (pCAG-GFP) or a KLF4 cDNA that is not targeted by miR-135s (pCAG-KLF4; van Erp et al., 2015). Overexpression of KLF4 did not affect RGC axon regeneration, but partly normalized the regeneration promoting effect of miR-135 mimic injection after ONI (0.2 mm, control-1 + GFP vs miR-135ab + KLF4: $46.89 \pm$ 6.816 vs $115.4 \pm 24.63, t_{(96)}=3.128, p=0.028$, one-way ANOVA with Sidak post hoc test; Fig. $6 D, E)$. Importantly, this effect of KLF4 was not due to its ability to regulate miR-135 expression because miR-135a and miR-135b levels in the retina were similar after miR-135ab + GFP and miR-135ab + KLF4 administration (Fig. 6F).

Intravitreal injection may target miRNA mimics to different cell types in the mouse retina. To ensure that miR-135a and miR135b can have a positive, cell-autonomous effect in RGCs on axon regeneration, overexpression of both miRNAs was induced by intravitreal injection of AAV2, a viral serotype known to target RGCs specifically (Fig. 6A, G; Weitz et al., 2013). Indeed, targeting miR-135s to RGCs induced RGC axon regeneration at a level comparable to that observed after mimic injection $(0.2 \mathrm{~mm}$, AAV2-control vs AAV2-miR-135ab: $53.76 \pm 10.62$ vs $243.6 \pm$ $23.59, t_{(30)}=10.98, p<0.0001$. one-way ANOVA with Sidak post hoc test; Fig. $6 \mathrm{H}$ ). Finally, to assess a potential endogenous role of miR-135a and miR-135b in regenerating RGC axons, specific miRNA sponges designed to sequester miR-135a and miR-135b were injected intravitreally. Decreased availability of the miRNAs resulted in a small but significant decrease in the number of regenerating axons close to the injury site compared with scrambled control sponge transfection $(0.2 \mathrm{~mm}$, control sponge vs $\mathrm{miR}-135 \mathrm{a} / \mathrm{b}$ sponge: $49.6 \pm 4.566$ vs $28.46 \pm 7.593, t_{(18)}=3.589$, $p=0.0063$. one-way ANOVA with Sidak post hoc test; Fig. $6 I$ ). Together, these results indicate that overexpression of miR-135 promotes CNS axon regeneration in part by reducing KLF4 expression, whereas decreasing functional miR-135 levels further reduces the regenerative potential of adult RGCs.

\section{Discussion}

miRNAs are highly abundant in neurons and have been linked to various aspects of neuronal development and function. However, the in vivo function(s) and downstream mRNA targets of many of the neuronally expressed miRNAs remain unknown. Here, we performed high-content screening of $>1000$ miRNAs in neuronal cells and identified miRNA-135a and miRNA-135b as potent stimulators of axon growth and neuron migration through the inhibition of the transcription factor KLF4. Further, although injured CNS axons generally lack significant regenerative capacity, our data show that repression of KLF4 by intravitreal application of miRNA-135 mimics promotes regeneration of RGC axons after ONI. In contrast, lowering miR-135 levels further inhibited RGC axon regeneration. Together, these results identify novel neuronal roles for the miRNA-135-KLF4 pathway and show that ectopic miRNA application to injured neurons can be used to enhance mammalian CNS axon regeneration.

\section{Novel neuronal functions for miR-135a and miR-135b}

The expression of members of the miR-135 family is regulated in specific types of cancer. miRNA-135s target many different tumor-related genes and thereby affect cell proliferation, differentiation, and metastasis formation (Nagel et al., 2008; Lin et al., 2013; Valeri et al., 2014; Ren et al., 2015; Shi et al., 2015). Because of their important role in cancer, both the diagnostic and thera- 
A

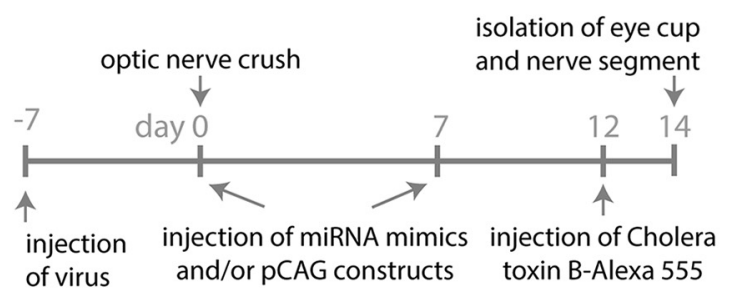

\section{B}

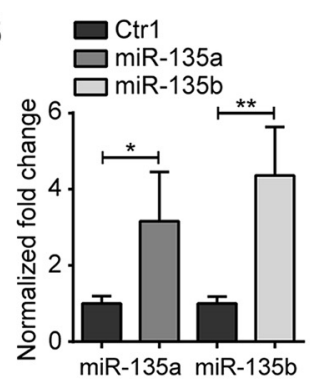

C
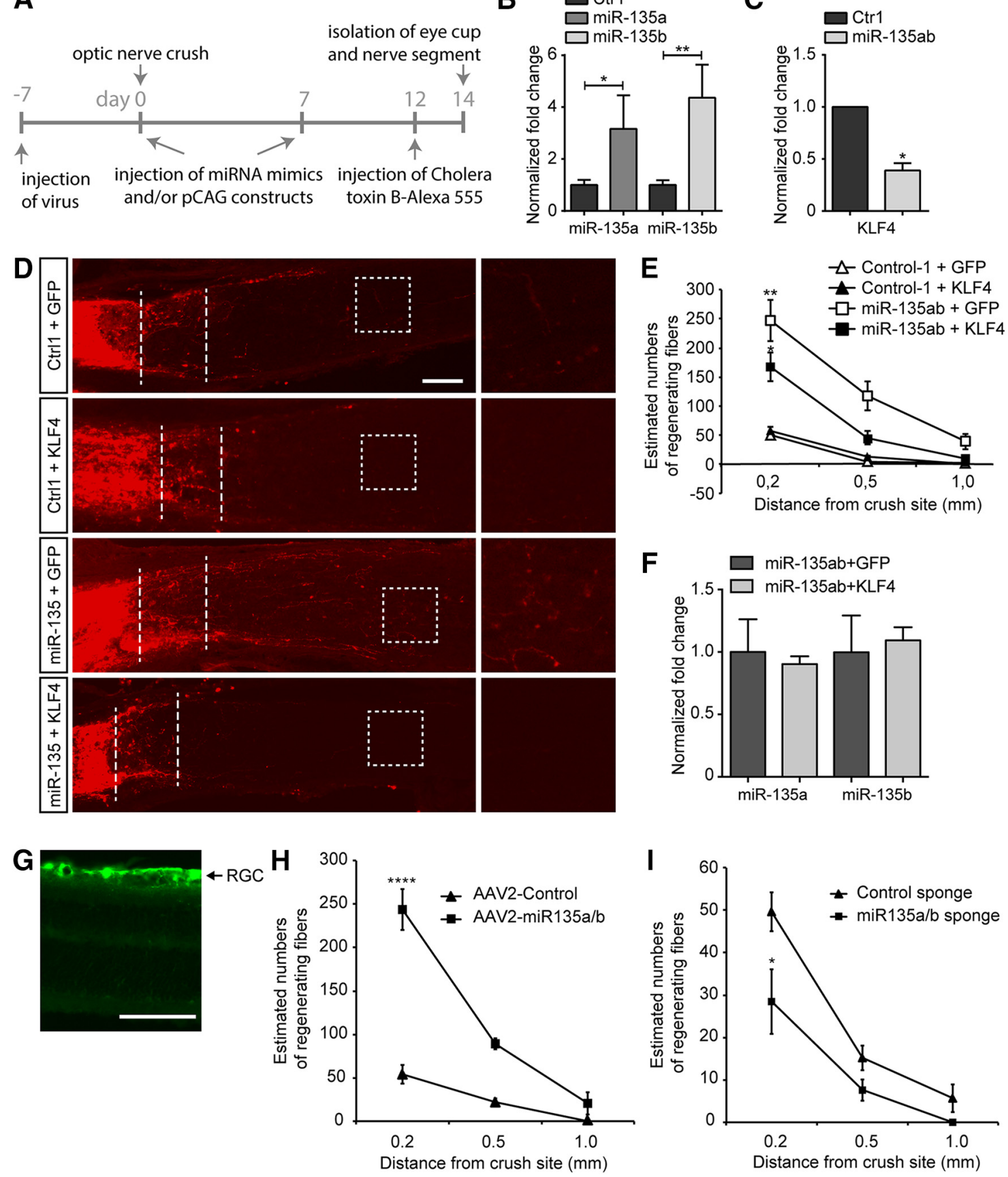

Figure 6. Exogenous miR-135s enhance axon regeneration after ONI. $A$, Experimental setup of the optic nerve crush studies. $B, C$, Graph shows results of qPCR on eye tissue after injection of mimics. miR-135a and miR-135b levels are increased after two injections of miRNA-mimics, whereas KLF4 levels are decreased ( $\boldsymbol{C}$. Fold changes are relative to $5 \mathrm{~S}$ housekeeping rRNA expression. Data are expressed as means \pm SEM. ${ }^{*} p<0.05,{ }^{* *} p<0.01, t$ test on delta Ct values. $\boldsymbol{D}$, Representative images of optic nerves stained for Cholera-toxin B conjugated to Alexa Fluor-555 $14 \mathrm{~d}$ after optic nerve crush. After injection of miR-135 mimics axons grow into and beyond the injury site (dotted lines indicate proximal and distal boundaries site of injury). Boxes indicate highermagnification images shown at the right. Scale bar, $100 \mu \mathrm{m}$. $\boldsymbol{E}$, Graph showing quantification of the number of regenerating axons relative to the distal end of the crush site at $14 \mathrm{~d}$ after injury for the conditions represented in $\boldsymbol{D} . n=9$ mice per condition. ${ }^{*} p<0.05$, ${ }^{* * *} p<0.0001$, ANOVA followed by Sidak's test. Data are presented as means \pm SEM. $\boldsymbol{F}$, Graph showing the results of qPCR on eye tissue after cotransfection of miR-135 mimics and GFP or KLF4 vector. No differences in miR-135a and miR-135b expression were seen between the GFP- or KLF4-transfected groups. Fold changes are relative to $5 S$ housekeeping rRNA expression. Data are expressed as means \pm SEM. G, AAV2-GFP virus was injected intravitreally. One week after injection, strong GFP signals are detected in RGCs but not in other cell types in the retina. Scale bar, $100 \mu \mathrm{m}$. $\boldsymbol{H}$, Graph showing quantification of the number of regenerating axons relative to the distal end of the crush site at $14 \mathrm{~d}$ after injury for experiments performed using intravitreal injection of AAV2 (at $7 \mathrm{~d}$ after lesion) expressing control miRNA or miR-135a and miR-135b (in addition to GFP). $n=6$ mice per condition. ${ }_{* * * *}^{*}<0.0001$, ANOVA followed by Sidak's test. Data are presented as means \pm SEM. I, Graph showing quantification of the number of regenerating axons relative to the distal end of the crush site at $14 \mathrm{~d}$ after injury for experiments performed using intravitreal injection of control or miR-135a and miR-135b sponge vectors. $n=6$ mice per condition. ${ }^{* *} p<0.01$, ANOVA followed by Sidak's test. Data are presented as means \pm SEM.

peutic use of miR-135s are being investigated intensely (Khatri and Subramanian, 2013; Wu et al., 2014; Zhang et al., 2016). Despite specific expression of miR-135s in the developing and adult nervous system (Fig. 2; Lagos-Quintana et al., 2001; Sem- pere et al., 2004; Ziats and Rennert, 2014; Caronia-Brown et al., 2016), much less is known about the neuronal functions of miR135s. miR-135a is required for chronic stress resiliency, antidepressant efficacy, and 5-HT activity in adult mice (Issler et al., 
2014) and may contribute to early mouse brain development (Caronia-Brown et al., 2016). At the cellular level, miR-135s mediate long-lasting spine remodeling in hippocampal synaptic LTD by targeting complexin-1 and complexin-2 (Hu et al., 2014). Previous work and our current results show developmentally regulated expression patterns for both miR-135a and miR-135b during late embryonic and postnatal development (Ziats and Rennert, 2014). However, the role of miR-135s at these developmental stages was unknown. Interestingly, miR-135b was the top hit of our image-based miRNA screen for neuronal morphology and subsequent experiments confirmed and extended this observation by revealing that neuronal miR-135b and its homolog miR-135a are required for neurite outgrowth and cortical neuron radial migration in vitro and in vivo. These observations reveal novel functions for miR-135a and miR-135b and are intriguing because miR-135a has been linked to disorders such as epilepsy and schizophrenia, which are characterized by structural changes in neuronal networks (Kan et al., 2012; Rossi et al., 2014; Alsharafi and Xiao, 2015). For example, expression of miR-135a is upregulated in the hippocampus of patients suffering from temporal lobe epilepsy (Kan et al., 2012) and it will be interesting to determine whether, and if so how, this miRNA contributes to pathogenic processes such as mossy fiber sprouting and granule cell dispersion observed during epilepsy.

\section{KLF4 is a downstream target of miR-135a and miR-135b in neurons}

In this study, we identified KLF4 as a target of miR-135a and miR-135b in neurons. KLF4 is a transcription factor that, apart from its function in self-renewal of embryonic stem cells, regulates the intrinsic neurite growth capacity of neurons (Moore et al., 2009; Steketee et al., 2014). Overexpression of KLF4 in RGCs strongly represses neurite outgrowth and branching. Conversely, downregulation of KLF4 induces longer neurites and enhances cortical neuron migration (Qin and Zhang, 2012; Fang et al., 2016). KLF4 is downregulated during neuronal differentiation and development and KLF4 overexpression impairs neural progenitor cell proliferation and differentiation (Qin et al., 2011). KLF4 expression gradually increases in mature neurons, correlating with a gradual loss in the ability of neurons to regrow their neurites (Moore et al., 2009; Blackmore et al., 2010). Several of our observations identify KLF4 as an important neuronal target of miR-135s. First, miR-135 mimics downregulate KLF4 expression in luciferase assays, neuronal cells, and RGCs in vivo. This is consistent with the ability of miR-135a to regulate KLF4 and KLF4-dependent STAT3 signaling negatively in non-neuronal cells (Lin et al., 2016; Yao et al., 2016). Second, KLF4 knock-down and miR-135 overexpression have similar effects on neuron migration, neurite growth, leading process length, and RGC axon regeneration. Third, the neurite (re-)growth and neuron migrationpromoting effects of miR-135 overexpression can be rescued by expression of miR-135-insensitive KLF4 constructs. Interestingly, whereas overexpression of KLF4 fully rescues the positive effects of miR-135s on cortical neuron migration, it only partially reverses the effects of these miRNAs on neurite growth and regeneration. It is possible that the levels of exogenous KLF4 expression were insufficient to reverse KLF4 expression to endogenous levels. Alternatively, because miRNAs are known to target multiple different mRNAs, it is possible that miR-135 mimics affect different transcripts related to neurite growth and migration other than KLF4. Indeed, in non-neuronal cells, miR-135a and miR-135b are known to target, for example, APC, GSK3 $\beta$, and FOXO1, all of which have been implicated in neurite growth or neuron migration (Nagel et al., 2008; Moritoki et al., 2014; Yang et al., 2015; and data not shown). Furthermore, KLF4 is part of a larger family of KLF proteins, several of which regulate axon growth (Apara and Goldberg, 2014). Bioinformatics analysis reveals that other family members, including KLF6, KLF8, KLF9, KLF13, and KLF16, have predicted binding sites for miR-135a and miR-135b in their $3^{\prime}$-UTRs (data not shown).

\section{Exogenous application of miR-135s enhances CNS axon regeneration}

The expression of miRNAs is strongly regulated after injury to the PNS and CNS. Knock-out of Dicer, a protein required for miRNA biogenesis, hampers peripheral nerve regeneration, whereas select miRNAs can function as intrinsic inhibitors of CNS axon regeneration (Song et al., 2012; Wu et al., 2012; Zou et al., 2013; Hancock et al., 2014; Phay et al., 2015; Li et al., 2016; Martirosyan et al., 2016). Consistent with these observations, manipulation of miRNA expression in mammalian adult sensory neurons promotes the regeneration of their axons in the PNS and CNS (Jiang et al., 2015; Gaudet et al., 2016; Hu et al., 2016). However, whether direct manipulation of miRNAs in injured mammalian CNS neurons also enhances axon regeneration and, if so, through which mRNA targets was unknown.

KLF4 binds STAT3 and represses the JAK-STAT3 pathway (Qin et al., 2013). Optic nerve crush experiments have shown that downregulation of KLF4 and concomitant activation of JAKSTAT3 signaling induces regenerative axon growth markedly in the hostile CNS environment (Moore et al., 2009; Qin et al., 2013). The fact that miR-135b was the top hit of our functional screen, together with the ability of miR-135b and miR-135a to promote axon growth through repression of KLF4, prompted us to explore whether miR-135s could be used to stimulate axon regrowth after CNS injury. Intravitreal administration of miR135 mimics to the retina after ONI reduced retinal KLF4 levels and triggered RGC axon regrowth into and across the lesion site. This effect of miR-135s was in part KLF dependent because reexpression of KLF4 in RGCs partially reversed the regrowth phenotype. Further, targeting miR-135s specifically to RGCs by viral vector-mediated delivery was sufficient to promote RGC axon regeneration. Previous studies have shown that miRNAs can be used to stimulate axon regeneration in the injured CNS (Jiang et al., 2015; Gaudet et al., 2016; Hu et al., 2016). However, our findings are conceptually distinct from these previous reports: we find that application of miRNAs to injured CNS neurons triggers axon regeneration after CNS injury, rather than regeneration of peripheral sensory axons in the CNS after delivery of miRNAs to the injury site. Therefore, whereas previous work has shown that miRNAs can be used to render the injured CNS more permissive for CNS axon regeneration, our work now shows that miRNAs can also be used to elevate the intrinsic growth potential of injured CNS neurons and thereby elicit regenerative growth. The ability of KLF4 reexpression to rescue the regeneration-promoting effects of miRNA-135s only partially supports the idea that these miRNAs target several different transcripts in the injured retina. miRNAmRNA binding only requires a $\sim 7-8 \mathrm{nt}$ match between miRNA and mRNA, so an individual miRNA can have dozens or possibly hundreds of targets. A single miRNA can regulate several genes in a single pathway or single genes in several pathways. This multitargeting property has obvious applications for promoting axon regeneration because it offers the means to disrupt several processes at once, a feature likely to be necessary for any future disease-modifying therapy. In addition, several different miRNAs may be combined into one therapy to target different cellular 
processes affecting regeneration; for example, glial scar formation and inflammation.

In conclusion, this study identifies miR-135a and miR-135b as novel regulators of intrinsic neurite outgrowth capacity and neuron migration by targeting KLF4. In addition, we find that exogenous application of miR-135 mimics to injured CNS neurons inhibits the intrinsic growth inhibitor KLF4 and thereby enhances CNS axon regeneration. Conversely, reducing miR-135 levels in the retina further decreased RGC axon regeneration. These data provide new tools for enhancing CNS axon regeneration and underscore the crucial role of these small noncoding RNAs after neural injury. Although most studies select miRNAs on basis of spatiotemporal expression or downstream targets, we have used high-content screening as an alternative and powerful strategy to identify novel neurite growth promoting miRNAs. Our screen identified several miRNAs with potent effects on neurite growth and branching. These included miRNAs with known effects on neurite development (e.g., miR-124), but also previously unexplored candidates (e.g., miR-220b). Further work is needed to establish firmly the function of these miRNAs and whether their aberrant expression or function is functionally linked to neural disorders such as epilepsy.

\section{References}

Aksoy-Aksel A, Zampa F, Schratt G (2014) MicroRNAs and synaptic plasticity-a mutual relationship. Philos Trans R Soc Lond B Biol Sci 369: pii: 20130515. CrossRef Medline

Alsharafi W, Xiao B (2015) Dynamic expression of MicroRNAs (183, 135a, $125 \mathrm{~b}, 128,30 \mathrm{c}$ and $27 \mathrm{a}$ ) in the rat pilocarpine model and temporal lobe epilepsy patients. CNS Neurol Disord Drug Targets 14:1096-1102. CrossRef Medline

Apara A, Goldberg JL (2014) Molecular mechanisms of the suppression of axon regeneration by KLF transcription factors. Neural Regen Res 9:1418-1421. CrossRef Medline

Baldwin KT, Giger RJ (2015) Insights into the physiological role of CNS regeneration inhibitors. Front Mol Neurosci 8:23. CrossRef Medline

Baudet ML, Bellon A, Holt CE (2013) Role of microRNAs in Semaphorin function and neural circuit formation. Semin Cell Dev Biol 24:146-155. CrossRef Medline

Blackmore MG, Moore DL, Smith RP, Goldberg JL, Bixby JL, Lemmon VP (2010) High-content screening of cortical neurons identifies novel regulators of axon growth. Mol Cell Neurosci 44:43-54. CrossRef Medline

Caronia-Brown G, Anderegg A, Awatramani R (2016) Expression and functional analysis of the Wnt/beta-catenin induced mir-135a-2 locus in embryonic forebrain development. Neural Dev 11:9. CrossRef Medline

Dickendesher TL, Baldwin KT, Mironova YA, Koriyama Y, Raiker SJ, Askew KL, Wood A, Geoffroy CG, Zheng B, Liepmann CD, Katagiri Y, Benowitz LI, Geller HM, Giger RJ (2012) NgR1 and NgR3 are receptors for chondroitin sulfate proteoglycans. Nat Neurosci 15:703-712. CrossRef Medline

Fang J, Shaw PX, Wang Y, Goldberg JL (2016) Kruppel-like factor 4 (KLF4) is not required for retinal cell differentiation. eNeuro 3.

Gaudet AD, Mandrekar-Colucci S, Hall JC, Sweet DR, Schmitt PJ, Xu X, Guan Z, Mo X, Guerau-de-Arellano M, Popovich PG (2016) miR-155 deletion in mice overcomes neuron-intrinsic and neuron-extrinsic barriers to spinal cord repair. J Neurosci 36:8516-8532. CrossRef Medline

Hancock ML, Preitner N, Quan J, Flanagan JG (2014) MicroRNA-132 is enriched in developing axons, locally regulates Rasal mRNA, and promotes axon extension. J Neurosci 34:66-78. CrossRef Medline

He Z, Jin Y (2016) Intrinsic control of axon regeneration. Neuron 90:437451. CrossRef Medline

Hu YW, Jiang JJ, Yan-Gao, Wang RY, Tu GJ (2016) MicroRNA-210 promotes sensory axon regeneration of adult mice in vivo and in vitro. Neurosci Lett 622:61-66. CrossRef Medline

Hu Z, Yu D, Gu QH, Yang Y, Tu K, Zhu J, Li Z (2014) miR-191 and miR-135 are required for long-lasting spine remodelling associated with synaptic long-term depression. Nat Commun 5:3263. CrossRef Medline

Issler O, Haramati S, Paul ED, Maeno H, Navon I, Zwang R, Gil S, Mayberg HS, Dunlop BW, Menke A, Awatramani R, Binder EB, Deneris ES, Lowry CA, Chen A (2014) MicroRNA 135 is essential for chronic stress resil- iency, antidepressant efficacy, and intact serotonergic activity. Neuron 83:344-360. CrossRef Medline

Jiang JJ, Liu CM, Zhang BY, Wang XW, Zhang M, Saijilafu, Zhang SR, Hall P, Hu YW, Zhou FQ (2015) MicroRNA-26a supports mammalian axon regeneration in vivo by suppressing GSK3 $\beta$ expression. Cell Death Dis 6:e1865. CrossRef Medline

Kan AA, van Erp S, Derijck AA, de Wit M, Hessel EV, O’Duibhir E, de Jager W, Van Rijen PC, Gosselaar PH, de Graan PN, Pasterkamp RJ (2012) Genome-wide microRNA profiling of human temporal lobe epilepsy identifies modulators of the immune response. Cell Mol Life Sci 69:31273145. CrossRef Medline

Khatri R, Subramanian S (2013) MicroRNA-135b and its circuitry networks as potential therapeutic targets in colon cancer. Front Oncol 3:268. CrossRef Medline

Lagos-Quintana M, Rauhut R, Lendeckel W, Tuschl T (2001) Identification of novel genes coding for small expressed RNAs. Science 294:853-858. CrossRef Medline

Lagos-Quintana M, Rauhut R, Yalcin A, Meyer J, Lendeckel W, Tuschl T (2002) Identification of tissue-specific microRNAs from mouse. Curr Biol 12:735-739. Medline

Li P, Teng ZQ, Liu CM (2016) Extrinsic and intrinsic regulation of axon regeneration by microRNAs after spinal cord injury. Neural Plast 2016: 1279051. Medline

Lin CW, Chang YL, Chang YC, Lin JC, Chen CC, Pan SH, Wu CT, Chen HY, Yang SC, Hong TM, Yang PC (2013) MicroRNA-135b promotes lung cancer metastasis by regulating multiple targets in the Hippo pathway and LZTS1. Nat Commun 4:1877. CrossRef Medline

Lin L, He Y, Xi BL, Zheng HC, Chen Q, Li J, Hu Y, Ye MH, Chen P, Qu Y (2016) miR-135a suppresses calcification in senescent VSMCs by regulating KLF4/STAT3 pathway. Curr Vasc Pharmacol 14:211-218. CrossRef Medline

Martirosyan NL, Carotenuto A, Patel AA, Kalani MY, Yagmurlu K, Lemole GM Jr, Preul MC, Theodore N (2016) The role of microRNA markers in the diagnosis, treatment, and outcome prediction of spinal cord injury. Front Surg 3:56. Medline

Moore DL, Blackmore MG, Hu Y, Kaestner KH, Bixby JL, Lemmon VP, Goldberg JL (2009) KLF family members regulate intrinsic axon regeneration ability. Science 326:298-301. CrossRef Medline

Moritoki Y, Hayashi Y, Mizuno K, Kamisawa H, Nishio H, Kurokawa S, Ugawa S, Kojima Y, Kohri K (2014) Expression profiling of microRNA in cryptorchid testes: miR-135a contributes to the maintenance of spermatogonial stem cells by regulating FoxO1. J Urol 191:1174-1180. CrossRef Medline

Nagel R, le Sage C, Diosdado B, van der Waal M, Oude Vrielink JA, Bolijn A, Meijer GA, Agami R (2008) Regulation of the adenomatous polyposis coli gene by the miR-135 family in colorectal cancer. Cancer Res 68:57955802. CrossRef Medline

Phay M, Kim HH, Yoo S (2015) Dynamic change and target prediction of axon-specific microRNAs in regenerating sciatic nerve. PLoS One 10: e0137461. CrossRef Medline

Poell JB, van Haastert RJ, Cerisoli F, Bolijn AS, Timmer LM, Diosdado-Calvo B, Meijer GA, van Puijenbroek AA, Berezikov E, Schaapveld RQ, Cuppen E (2011) Functional microRNA screening using a comprehensive lentiviral human microRNA expression library. BMC Genomics 12:546. CrossRef Medline

Qin S, Zhang CL (2012) Role of Kruppel-like factor 4 in neurogenesis and radial neuronal migration in the developing cerebral cortex. Mol Cell Biol 32:4297-4305. CrossRef Medline

Qin S, Liu M, Niu W, Zhang CL (2011) Dysregulation of Kruppel-like factor 4 during brain development leads to hydrocephalus in mice. Proc Natl Acad Sci U S A 108:21117-21121. CrossRef Medline

Qin S, Zou Y, Zhang CL (2013) Cross-talk between KLF4 and STAT3 regulates axon regeneration. Nat Commun 4:2633. CrossRef Medline

Ren JW, Li ZJ, Tu C (2015) miR-135 post-transcriptionally regulates FOXO1 expression and promotes cell proliferation in human malignant melanoma cells. Int J Clin Exp Pathol 8:6356-6366. Medline

Rossi M, Kilpinen H, Muona M, Surakka I, Ingle C, Lahtinen J, Hennah W, Ripatti S, Hovatta I (2014) Allele-specific regulation of DISC1 expression by miR-135b-5p. Eur J Hum Genet 22:840-843. CrossRef Medline

Sempere LF, Freemantle S, Pitha-Rowe I, Moss E, Dmitrovsky E, Ambros V (2004) Expression profiling of mammalian microRNAs uncovers a sub- 
set of brain-expressed microRNAs with possible roles in murine and human neuronal differentiation. Genome Biol 5:R13. CrossRef Medline

Shi H, Ji Y, Zhang D, Liu Y, Fang P (2015) miR-135a inhibits migration and invasion and regulates EMT-related marker genes by targeting KLF8 in lung cancer cells. Biochem Biophys Res Commun 465:125-130. CrossRef Medline

Song Y, Ori-McKenney KM, Zheng Y, Han C, Jan LY, Jan YN (2012) Regeneration of Drosophila sensory neuron axons and dendrites is regulated by the Akt pathway involving Pten and microRNA bantam. Genes Dev 26:1612-1625. CrossRef Medline

Steketee MB, Oboudiyat C, Daneman R, Trakhtenberg E, Lamoureux P, Weinstein JE, Heidemann S, Barres BA, Goldberg JL (2014) Regulation of intrinsic axon growth ability at retinal ganglion cell growth cones. Invest Opthalmol Vis Sci 55:4369-4377. CrossRef Medline

Valeri N, et al. (2014) MicroRNA-135b promotes cancer progression by acting as a downstream effector of oncogenic pathways in colon cancer. Cancer Cell 25:469-483. CrossRef Medline

Van Battum EY, Gunput RA, Lemstra S, Groen EJ, Yu KL, Adolfs Y, Zhou Y, Hoogenraad CC, Yoshida Y, Schachner M, Akhmanova A, Pasterkamp RJ (2014) The intracellular redox protein MICAL-1 regulates the development of hippocampal mossy fibre connections. Nat Commun 5:4317. CrossRef Medline

van Erp S, van den Heuvel DM, Fujita Y, Robinson RA, Hellemons AJ, Adolfs Y, Van Battum EY, Blokhuis AM, Kuijpers M, Demmers JA, Hedman H, Hoogenraad CC, Siebold C, Yamashita T, Pasterkamp RJ (2015) Lrig2 negatively regulates ectodomain shedding of axon guidance receptors by ADAM proteases. Dev Cell 35:537-552. CrossRef Medline

Weitz AC, Behrend MR, Lee NS, Klein RL, Chiodo VA, Hauswirth WW, Humayun MS, Weiland JD, Chow RH (2013) Imaging the response of the retina to electrical stimulation with genetically encoded calcium indicators. J Neurophysiol 109:1979-1988. CrossRef Medline

Wu CW, Ng SC, Dong Y, Tian L, Ng SS, Leung WW, Law WT, Yau TO, Chan FK, Sung JJ, Yu J (2014) Identification of microRNA-135b in stool as a potential noninvasive biomarker for colorectal cancer and adenoma. Clin Cancer Res 20:2994-3002. CrossRef Medline

Wu D, Raafat A, Pak E, Clemens S, Murashov AK (2012) Dicer-microRNA pathway is critical for peripheral nerve regeneration and functional recovery in vivo and regenerative axonogenesis in vitro. Exp Neurol 233:555565. CrossRef Medline

Xiao F, Zuo Z, Cai G, Kang S, Gao X, Li T (2009) miRecords: an integrated resource for microRNA-target interactions. Nucleic Acids Res 37:D105D110. CrossRef Medline

Yang X, Wang X, Nie F, Liu T, Yu X, Wang H, Li Q, Peng R, Mao Z, Zhou Q, Li G (2015) miR-135 family members mediate podocyte injury through the activation of Wnt/ $\beta$-catenin signaling. Int J Mol Med 36:669-677. CrossRef Medline

Yao S, Tian C, Ding Y, Ye Q, Gao Y, Yang N, Li Q (2016) Down-regulation of Krüppel-like factor-4 by microRNA-135a-5p promotes proliferation and metastasis in hepatocellular carcinoma by transforming growth factor- $\beta 1$. Oncotarget 7:42566-42578. CrossRef Medline

Yau KW, van Beuningen SF, Cunha-Ferreira I, Cloin BM, van Battum EY, Will L, Schätzle P, Tas RP, van Krugten J, Katrukha EA, Jiang K, Wulf PS, Mikhaylova M, Harterink M, Pasterkamp RJ, Akhmanova A, Kapitein LC, Hoogenraad CC (2014) Microtubule minus-end binding protein CAMSAP2 controls axon specification and dendrite development. Neuron 82:1058-1073. CrossRef Medline

Zhang YK, Sun B, Sui G (2016) Serum microRNA-135a downregulation as a prognostic marker of non-small cell lung cancer. Genet Mol Res 15.

Ziats MN, Rennert OM (2014) Identification of differentially expressed microRNAs across the developing human brain. Mol Psychiatry 19:848852. CrossRef Medline

Zou Y, Chiu H, Zinovyeva A, Ambros V, Chuang CF, Chang C (2013) Developmental decline in neuronal regeneration by the progressive change of two intrinsic timers. Science 340:372-376. CrossRef Medline 